A systematic literature review of assessment feedback in Preclinical Dental Education

Andrea Rung: School of Dentistry and Oral Health, Griffith University, a.rung@griffith.edu.au,

Roy George: School of Dentistry and Oral Health, Griffith University

drroygeorge@gmail.com

Griffith University, Gold Coast Campus, Griffith Health Centre (G40) c/o Olsen Av \& Parkland Dr

This article has been accepted for publication and undergone full peer review but has not been through the copyediting, typesetting, pagination and proofreading process, which may lead to differences between this version and the Version of Record. Please cite this article as doi: 10.1111/EJE.12584

This article is protected by copyright. All rights reserved 
DR. ANDREA RUNG (Orcid ID : 0000-0003-3469-3629)

Article type : Original Article

\section{A systematic literature review of assessment feedback in Preclinical Dental Education}

\section{Abstract:}

Introduction: Development of evaluative judgement abilities through assessment and feedback strategies are crucial in students' progress to become self-regulated life-long learners. Dentists ability to appraise the quality of treatment provided to their patients starts with development of competences in a simulated preclinical training environment. However, the effectiveness of these assessment and feedback strategies is not well understood.

Material and methods: This literature review aims to identify assessment and feedback interventions and their effect on dental students' appraisal and psychomotor skills during preclinical training. A search of the electronic databases PubMed, Ebscohost (Dentistry and Oral Health), Scopus, Web of Science and EMBASE was conducted of studies published until October 2019.

Results: Twelve studies met the inclusion criteria. Two studies were randomised controlled trials and ten non-randomised studies. All studies included students' self-assessment and grading criteria as part of their feedback interventions. Feedback utilisation training was reported in $66 \%$ of the included studies. Evidence suggests feedback strategies improve psychomotor skills and increase agreement between students and teachers awarded grades. However, these improvements vary with the complexity of the task. Only one study shows self-directed learning significantly contributing to students' psychomotor skills improvement. Conclusion: Although assessment and feedback are regarded as crucial for developing students' evaluative judgement capabilities to become self-regulated life-long learners, there is limited empirical evidence of the assessment and feedback conditions that enhance students judgement abilities, performance and self regulation. Further research is needed to investigate and compare the effect of different assessment and feedback interventions on students' appraisal skills, their impact on learning and self-regulation. 
Key words: assessment, feedback, evaluation, learning, preclinical, psychomotor, selfregulation 


\section{Introduction}

Assessment and feedback are reported to be crucial for developing students' judgement abilities that are essential to become self-regulated life-long learners ${ }^{1}$. Self-regulation is a context dependent attribute that can be developed in a constructive process and constrained by features of the learning environment ${ }^{2-4}$. Self regulation is important for two main reasons: A) It empowers students to take control over their learning making them 'more persistent, resourceful, confident and higher achievers ${ }^{, 2}$. B) It allows learners adapt to changes and maintain competency, an attribute expected of health professionals ${ }^{5-7}$.

There are numerous definitions of assessment depending on the context, functions and aims ${ }^{8}$. In the context of this study assessment refers to the appraisal of students work that generates information used to improve learning and teaching: Formative assessments ${ }^{9}$. A crucial aspect of formative assessment is feedback. Feedback is often used as a synonym for information passed from the instructor to the student. Although, there is no consensus in the literature for the definition of feedback ${ }^{10}$, it is agreed that feedback supports learning when students actively engage in the learning process and have opportunities to apply the information gained from it ${ }^{11} 12$. Therefore, feedback could be defined as a dialogue between learner and instructor or peers where the learner not only receives comments (advice, criticism, opinion) but also pursues it; this process is not the end point but rather the beginning of learning .

It is further argued that assessment and feedback strategies support students independent and effective learning when they explicitly develop students' evaluative judgement ${ }^{13}$. Evaluative judgement is the "capability to make decisions about quality of work of self and others" 14 and it underpins learners' ability to engage with feedback from multiple sources within and beyond university courses enhancing learners' ability to adapt and change tactics and behaviours $^{14}$.

In the context of dentistry, the simulated clinical environment is where dental students begin to develop their psychomotor and appraisal skills that will enable learners to self-regulate. May et al. ${ }^{15}$ argue that self-assessment should be systematically taught to students because accurate self-assessment is an indispensable skill for dental practitioners to become life-long learners. Indeed, self-assessment activities are reported to enable learners to identify goals, recognise their current level of achievement and develop strategies to achieve these goals $9,16,17$. Self-assessment activities are an essential aspect of any formative assessment because 
it provides students with the opportunity to reflect on the quality of their work and learn in the process ${ }^{18}$.

Emerging literature in higher education, however, defines self-assessment not as a skill but as pedagogical strategy, along with feedback, rubrics, exemplars, and peer assessment, that can be adapted to exercise and develop students evaluative judgement. ${ }^{19}$ This dissonant definition lead us to investigate what assessment feedback strategies are being used in preclinical dentistry to enhance students' evaluative judgement.

There is currently a large body of literature on assessment and feedback in higher education in which dental education is underrepresented ${ }^{8,10,20,21}$. Furthermore, to our knowledge, there are no reviews of assessment and feedback interventions used in dentistry. An improved understanding of feedback interventions used in pre-clinical dentistry is needed to inform educators and academics interested in developing feedback strategies that assist students learning. To this end, a systematic review of the literature will identify the utilisation and efficacy of assessment and feedback interventions in preclinical dentistry and their impact on students' psychomotor skills learning.

The objectives of this study are:

1. To determine feedback interventions used in preclinical dentistry.

2. To describe the characteristics of the interventions.

3. To find the outcomes of the feedback interventions 


\section{Methodology:}

This systematic literature review followed the "Preferred Reporting Items for Systematic Reviews and Meta-Analysis" (PRISMA) guidelines ${ }^{22}$. The database used for this study were: MEDLINE (PubMed Interface), Ebscohost (Dentistry and Oral Health), Scopus, Web of Science, EMBASE. To time limit was set for the search up to October 2019. The focus search criteria or combination used were: Assessment, evaluation, feedback, dentistry, dental, education [Table 1].

The review used the PICO (Population, Intervention, Comparator and Outcome) to guide the criteria for inclusion and exclusion of studies. The population $(\mathrm{P})$ included dental students in preclinical courses. Interventions ( I ) were all feedback exchanges generated within assessment of psychomotor skills, occurring within and beyond the immediate learning context, being overt or covert (actively and/or passively sought and/or received), and drawing from a range of resources ${ }^{10}$. The comparisons (C) were between groups, intervention and control, and within same group overtime. The primary outcome $(\mathrm{O})$ measured was students' psychomotor skills while the secondary outcome was students' appraisal skills.

\section{Selection procedure:}

One author $(\mathrm{xx})$ identified all titles and abstracts and removed the duplicates. After screening the abstracts and titles, inappropriate articles were removed based on the PICO inclusion criteria. The remaining articles were evaluated following the eligibility. The second author independently reviewed the remaining full text versions (xy). Disagreements between authors were resolved by consensus. The results of the screening process are shown in Figure 1 flow diagram. ${ }^{23}$

\section{Eligibility criteria:}

All articles on assessment feedback interventions in preclinical dental education. Inclusion criteria:

- Settings: Dental schools

- Participants: Dental students and teaching staff

- Study design: Randomised control trials and non-randomised studies.

- Language: English 
- Year: Articles published up until October 2019

\section{Exclusion criteria:}

- Technology feedback: Studies with feedback provided by other means than human judgement e.g. haptic, scanners.

- Studies in clinical settings

- Assessment feedback interventions on aspects other than psychomotor skills. For example, knowledge

- Studies without students involved such faculty calibration studies.

- Theory: Publications describing assessment feedback methods

- Type of study: Descriptive studies without comparisons.

\section{Data extraction and analysis}

1. Study design features and risk of bias. There is ambiguity in the labels asigned to study design features. Consequently, the relevant data about study design features were collected using the Cochrane checkllist for systematic reviews of nonrandomised studies [Table 2] ${ }^{24}$. The check list further assisted the authors of this review to decide the elegibility of the studies. Risk of bias for randomised and not randomised studies were undertaken separatedly as recommended by the Cochrane guidelines ${ }^{24}$. For the randomised control trials the Cochrane risk of bias tool was used [Figure 2] and a different tool for non-randomised studies ${ }^{25}$ [Table 3]. No metaanalysis was undertaken because of the variety of interventions, study designs and type of measurements.

2. Feedback interventions components were classified and organised using Winston and colleagues' categories ${ }^{8}$ [Table 4]. Descriptions of each components are Appendix 1.

3. The description of educational interventions was done collecting and collating data using a modified table from a publication on characteristics of educational interventions [Table 5] ${ }^{26}$. 


\section{Results:}

Twelve studies met the inclusion criteria. All studies were published in dental or dental education journals. Nine in the Journal of Dental Education, two in the European Journal of Dental Education, one in the International Endodontic Journal.

\section{Studies designs features}

There were two main types of study: A) Studies comparing an intervention with a control group: Two randomised control trials (RCT) ${ }^{27,28}$, three non-randomised studies (NRS) ${ }^{29-31}$ and B) Studies comparing intervention outcomes within the same group overtime: Eight NRS 31-37 38. [Table 1]. One prospective three arm study ${ }^{30}$, compared an intervention with a control group and within the same group overtime.

\section{Risk of bias within studies}

All studies presented risk of bias in at least one domain. Notably, blinding participants to the study as this is often a domain impossible to control in learning settings ${ }^{30-32,34,35,37}$. Students' previous skills or academic abilities ${ }^{31-33,37,38}$ and measurement exposure ${ }^{31,35}$ was the second most uncontrolled element of the studies [Figure 2].

\section{Feedback interventions}

Following Winston and colleagues' classification ${ }^{8}$, feedback components were organised in four groups based on their conceptual similarities: 1) Internalising and applying standards, 2) Sustainable monitoring, 3) Collective provision of training and 4) Manner of feedback delivery. It is important to note that feedback components are not mutually exclusive and many of them are concurrently implemented [Table 3].

\section{Internalising and applying standards}

Feedback components in this group provide students with opportunities to become familiar with criteria standards and the process of appraising their own work and others ${ }^{8}$. 
Engaging with grading criteria: All studies reported using grading criteria in assisting students to recognize standards of quality during the appraisal process. Ten studies use criteria developed by teachers to produce a final grade by adding individual criterion's scores $^{27-30,32,33,35-38}$. Two studies allowed more flexibility. One study permitted teachers and students to award half marks or override the score ${ }^{34}$ while in another study the criteria was created in collaboration with the students ${ }^{31}$.

Details on the validation and development of criteria vary from providing thorough details of the process to only making reference of their use ${ }^{28,35}$. Huth et $\mathrm{al}^{30}$ described the matrix analysis and criterion weighting worked out by collaborative efforts of four dental schools ${ }^{30}$. De Peralta et $\mathrm{al}^{37}$, report a similar collaborative and collegial participation in reviewing and updating of marking criteria used in their study ${ }^{37}$. Majority of studies only displayed marking criteria rubrics with no reference to their development or validation ${ }^{27,29,30,32-34,36-38}$.

Information on graders reliability using the marking criteria is also reported with different amount of detail. Some studies report a numerical value of reliability among graders 29,30,33,34,36,39 while others mention the calibrating strategies used by the graders ${ }^{35,37,38}$. [Table 4]

Self-assessment: All twelve studies included self-assessment as a component of the feedback intervention. Ten studies report students' self-assessment ability as changes in congruence between students' self-awarded grades and teachers' awarded grades ${ }^{27-30,32-37}$. Two studies report students access to grading forms and self-assessment as component of the feedback intervention but without recording grades ${ }^{38} 31$.

Peer assessment: Five studies reported students assessing peers' work as another source of feedback playing the role of promoting students' reflection and dialogue ${ }^{29,34-37}$. When implemented, peer assessment appears secondary to self-assessment and the two studies that measured congruence between peer and teachers grades found students tend to overestimate their peers ${ }^{34,35}$.

Dialogue and discussion: Dialogue and discussion support learners' goal setting, selfregulation and promote engagement with feedback ${ }^{8}$. Seven studies included dialogue and discussion. Three studies used only dialogue and discussion to improve students' 
understanding of the marking criteria and standards ${ }^{28,29,36}$. Interestingly, dialogue was also used among supervisors in calibration activities before assessing students work ${ }^{30,38}$. De Peralta et al ${ }^{37}$. used dialogue and discussion with supervisors to help students generate their own study plan. Manogue et $\mathrm{al}^{31}$. used dialogue among teachers and students to develop the criteria used to appraise students work.

\section{Sustainable monitoring}

This aspect of feedback allow students to record, monitor, and reflect on their performance and appraisal skills overtime ${ }^{8}$.

Action planning: Encouraging students to keep record of their progress is reported by Winstone et $\mathrm{al}^{8}$ to increase their engagement. Writing a self-generated study plan based on feedback from peer and instructors was used by students learning caries excavation ${ }^{37}$. In two studies, learners had to write corrective actions after comparing their own work with exemplars of different quality ${ }^{29,36}$.

\section{Collective provision of training}

This section includes strategies that provide group of students training and resources about the effective use feedback ${ }^{8}$. Although this training includes preparing students for their emotional response to feedback, this aspect was not present in any of the selected papers.

Feedback workshops: The description of students training to use and receive feedback vary in the level of detail provided. In one study, a teacher instructed students how to use the criteria $^{35}$. Three studies combined instructors' transmission and student active participation in dialogue with peer and supervisors to understand the marking criteria and practice how to use it. ${ }^{29,3627}$.

Exemplar assignments: The use of exemplars was aimed to help students understand the appraisal process. Three studies used photography and models of exemplars of different quality $^{27,29,36}$, another study utilised models only ${ }^{30}$ and a fifth study used video clips to facilitate dialogue and discussion between students and teachers ${ }^{28}$.

\section{Manner of feedback delivery}

This section refers to the mode of feedback delivery and its function ${ }^{8}$. 
Formative assessment: The purpose of all selected studies was to generate feedback. Five studies have a summative component at the end of the intervention to evaluate its effect on students' performance ${ }^{33-35,37,38}$. One study awarded students summative grades for selfassessment as an incentive to self-assess accurately ${ }^{33}$.

Feedback without a grade: In only one intervention feedback did not have a numerical or scale grade ${ }^{31}$.

Tailored feedback: This component refers to students selecting the aspect of their work they would like to have feedback on. In one study, students could select which one of three amalgam preparations they wanted instructors to provide feedback ${ }^{27}$. All other studies may have tailored their feedback, but this is not specified in the description of their methods.

\section{Interventions outcomes:}

Five interventions resulted in improved psychomotor skills and improved agreement between students and teachers' marks 323537 29,36. A study found similar results for two surfaces amalgam preparations but no differences between intervention and control group in simple occlusal preparations for amalgam ${ }^{27}$. Similarly, Huth et $\mathrm{al}^{30}$ did not find significant differences on performance or agreement of student-tutor marks except in complex tasks where more intense feedback training resulted in significantly closer agreement between self and instructor grading. Shah's intervention, on the other hand, enhanced students amalgam preparations but did not resulted in significant differences of self-assessment abilities between the intervention and control group ${ }^{28}$. Self-assessment scores were reported to be better predictor of students' performance than their teachers' scores by two studies ${ }^{33}$ even after one of the studies corrected for regressions towards the mean ${ }^{34}$.

Two studies did not measure the congruency between students and teacher grades. However, one study found that self-directed projects significantly contributed to students wax carving improvement ${ }^{38}$ and another reported increased student confidence after implementing a feedback strategy that required students self-assessment even when preclinical practice time was reduced ${ }^{31}$. In the later study, student performance was not measured. However, instructors reported students' performance was no different than before the intervention was implemented. 


\section{Discussion:}

A limited number of studies were selected despite the ubiquitous use of assessment feedback in preclinical dental education. Even more surprising is the lack of reference to the use of feedback in preclinical dentistry in the broad higher education literature ${ }^{8,10,20}$. This shows paucity of studies about assessment and feedback practices in preclinical dental education and the limited contribution of dental education into the broader higher education literature.

All selected studies reported using formative assessment strategies that included students internalising and applying standards by self-assessment and engagement with criteria standards as part of their feedback strategy. Not surprisingly self-assessment is central to all the feedback strategies. This reflective activity improves students' understanding of the appraisal process, ability to recognise and discriminate the quality of their work ${ }^{27,29,36}$ and aligns students and instructors objectives ${ }^{28}$. Hence, students able to recognise quality, or lack of it, are more likely to improve their performance when reattempting the task. ${ }^{33,34}$.

Changes in students' ability to judge the quality of their work is quantified as changes in congruence between students self-awarded grades and teachers' grades. Consequently, improvements in judgement ability are reported as agreement between students' self and teachers awarded grades ${ }^{27-30,32-37}$. This indicator of judgement ability is problematic because of the low inter-rater reliability among instructors ${ }^{40}$, underreported graders calibration ${ }^{18}$ and graders unconscious bias and heuristics in the appraisal process ${ }^{1}$.

Quantifying this ability can be further clouded if regression towards the mean (RTM) is not controlled. Regression towards the mean is a statistical phenomenon where unusually large or small measurements are followed by measures close to the mean. This occurs when repeated observations are done in the same subject or unit ${ }^{41}$. Consequently, changes in measurements caused by this statistical phenomenon can be incorrectly interpreted as the result of an intervention. Furthermore, it is important to note that eight studies compare their outcomes to base line [Table 2]. Statistical testing against baseline is common in dental research. However, comparison to baseline studies overall have been criticized for presenting false positive results ${ }^{42}$.

Engaging with detailed grading criteria appears to solve many of the self-assessment issues by allowing analytic grading. This makes the appraisal process more transparent and fair to 
students, and objective and accurate to teachers ${ }^{43,44}$. Analytic grading breaks a complex task into single components and then sums them to a single final grade. This, as the name implies, allows analysis of supervisors' grading consistency and students' progress. Analytic grading, however, present several challenges ${ }^{45}$. First, the sum of each criterion might not be a clear description of work quality since criteria boundaries might not be discrete and overlap. A criterion may be more important than others and, in more complex work, quality might be identified by an expert, the teacher, but not easily described in the criteria ${ }^{45}$. Only two studies appear to put a strategy in place to address this limitation of analytic grading. Chambers et $\mathrm{al}^{34}$ allowed students and teachers to override the grades according to their judgment and Geissler ${ }^{32}$ identifying the need for students to use their judgements to identify aspects of their work quality that are more important than others and hence carry more weight in the overall grade ${ }^{32}$. A second challenge is that grades in the form of a number, percentage or letter may be so encoded or cryptic that they become meaningless to students ${ }^{46}$. And while student self-awarded grades may match with the their teachers, they may convey little about students' work quality or judgement abilities ${ }^{46,47}$. Finally, criteria are open to interpretation and influenced by teachers' previous experience in the discipline, teaching environment and teachers marking experience ${ }^{40,48}$.

The limitations of self-assessment and analytic grading should not discourage educators from implementing and measuring their interventions but rather make these problematic aspects of the appraisal process explicit to students and teachers. For instance, dialogue and discussion between learners and teachers can improve understanding of the grading criteria and its use ${ }^{30,32}$ and help them to plan strategies to improve future performance ${ }^{37}$. More importantly, it will promote students' engagement with the feedback process ${ }^{8}$. Discussion and dialogue around one's own performance requires an environment of trust without fear of admitting mistakes $^{30,33}$ and a teachers' culture of students actively involved in the feedback process through discussion and reflection ${ }^{31}$. This environment and culture can be nourished by explicitly training learners, and teachers, to understand the feedback process and the role they play in it $^{8}$.

The information found in this review on assessment and feedback training varies from formally instructing students on the grading process to a more active involvement of students in the grading and feedback process. Notably by using exemplars. Judging the quality of exemplars gives a point of reference for meaningful feedback ${ }^{49}$ because students can 
experience and understand how instructors, experts, use criteria. This allows students to determine standards before their own work is assessed ${ }^{50,51},{ }^{27,29,30,36}$. This is the reason assessing exemplars is also known as a 'feedforward' ${ }^{52}$. Exposing students to examples alone may not enhance learning because students, particularly novice ones, may be unable to identify what is important or what is a good or poor work ${ }^{53}$. It is in the dialogue with tutors and peers that students can build an understanding of what quality is, particularly when this understanding requires a tacit knowledge that cannot be captured in criteria ${ }^{54}$. Dialogue around appraising exemplars also creates a community of practice whose participants will eventually share the same language and practices facilitating not only the acquisition of tacit knowledge and the interpretation of work quality but also strategies to achieve it ${ }^{53}$.

Learners need to understand terminology and concepts used in feedback for it to be effective 8 . However, there is little mention on the relation between students ability to articulate technical information and their evaluative judgement ability and performance ${ }^{36}$. Only one study reported on a feedback intervention where students wrote down an action plan with strategies to achieve the learning goals after engaging in dialogue with peer and teachers ${ }^{37}$. The ability of students and teachers to articulate and communicate feedback and its relation with learning is underreported perhaps because of difficulties in capturing students' and teachers justification for their grades and strategies ${ }^{37}$. This is a poorly understood aspect of feedback, one that could be facilitated by Information Communication technology (ICT).

Student self-regulation is another underreported aspect of feedback. Students' ability to assess their own strength and weakness as a learner is an essential aspect of self-regulation ${ }^{8}$. However, there is only one study reporting increased confidence among students actively engaged in self-assessment ${ }^{31}$. Despite the claims that assessment and feedback have a key role in developing learners independent learning, this review found little empirical evidence that this is the case. Except for Radjaeipur et al. ${ }^{38}$ who found that supplementary self-directed projects, without instructors' feedback, significantly counted for summative assessment improvements ${ }^{38}$ demonstrating that students as early as first year, provided the right conditions, are capable to regulate their learning and thrive.

Although, it should be acknowledged that further research is needed to understand the effect of different assessment and feedback interventions on students' learning, this study recommends: a) Use marking criteria to facilitate dialogue among students and between 
students and instructors. b) Implement self-assessment activities that encourages students to judge the quality of their work (what) and justify their judgement (why) rather than matching their grades with their peers and graders. c) Allocate time for dialogue between instructors and students where students can articulate, verbally or written, their evaluative judgement. d) Make instructors and students aware of aspects that interfere with human judgement such as unconscious bias and heuristics. e) Create the right conditions for students to regulate their own learning.

\section{Conclusion:}

Assessment and feedback are regarded crucial for developing students' evaluative judgement abilities to become self-regulated life-long learners. However, there is limited empirical evidence of the assessment and feedback conditions that enhance students evaluative judgement abilities, performance and self regualtion and hence further research is needed.

This article is protected by copyright. All rights reserved 


\section{References}

1. Joughin G, Boud D, Dawson P. Threats to student evaluative judgement and their management. High Educ Res Dev. 2018:1-13.

2. Nicol DJ, Macfarlane-Dick D. Formative assessment and self-regulated learning: A model and seven principles of good feedback practice. Stud High Educ. 2006;31(2):199-218.

3. Pintrich PR, Zusho A. Student motivation and self-regulated learning in the college classroom. In: The scholarship of teaching and learning in higher education: An evidence-based perspective. Springer; 2007:731-810.

4. Zimmerman BJ. Theories of self-regulated learning and academic achievement: An overview and analysis In: Zimmerman BJ, Schunk DH, eds. Self-regulated learning and academic achievement: Theoretical perspectives. Taylor \& Francis; 2013:1-35.

5. Australian Dental Council. Professional Competencies of a Newly Qualified Dentist. http://www.adc.org.au/documents/Professional Published 2016. Accessed 2017-0228.

6. Commission on Dental Accreditation. Accreditation Standards for Dental Education Programs. CODA. https://www.ada.org/ /media/CODA/Files/2020_pde.pdf?la=en. Published 2019. Accessed 16/4/2019, 2019.

7. Council of European Dentists. Competencies required for the practice of dentistry in the European Union. file://Users/s2125071/Downloads/CED-DOC-2009-026-EFIN.pdf. Published 2009. Accessed 28-2-2017, 2017.

8. Winstone NE, Nash RA, Parker M, Rowntree J. Supporting learners' agentic engagement with feedback: A systematic review and a taxonomy of recipience processes. Educ Psychol. 2017;52(1):17-37.

9. Sadler DR. Formative assessment and the design of instructional systems. Instructional science. 1989;18(2):119-144.

10. Evans C. Making sense of assessment feedback in higher education. Review of educational research. 2013;83(1):70-120.

11. Taras M. Feedback on feedback. In: Merry S, Price M, Carless D, Taras M, eds. Reconceptualising Feedback in Higer Education: Developing dialogue with students. London: Routledge; 2013. 
12. Boud D, Molloy E. Rethinking models of feedback for learning: the challenge of design. Assess Eval High Edu. 2013;38(6):698-712.

13. Carless D. Sustainable feedback and the development of students self-evaluative capacities. In: Merry S, Price M, Carless D, Taras M, eds. Resconseptualising Feedback in Higher Education: Developing dialogue with students. Taylor and Francis; 2013.

14. Tai J, Ajjawi R, Boud D, Dawson P, Panadero E. Developing evaluative judgement: enabling students to make decisions about the quality of work. High Educ. 2017;76(3):1-15.

15. Mays KA, Levine E. Dental Students' Self-Assessment of Operative Preparations Using CAD/CAM: A Preliminary Analysis. J Dent Educ. 2014;78(12):1673-1680.

16. Black P, Wiliam D. Inside the black box raising standards through classroom assessment. The Phi Delta Kappan 1998;80(2):139-144.

17. Black P, Wiliam D. Assessment and classroom learning. Assessment in Education. 1998;5(1):7-74.

18. Mays KA, Branch-Mays GL. A Systematic Review of the Use of Self-Assessment in Preclinical and Clinical Dental Education. J Dent Educ. 2016;80(8):902-913.

19. Ajjawi R, Tai J, Dawson P, Boud D. Conceptualising evaluative judgement for sustainable assessment in higher education. In: Boud D, Ajjawi R, Dawson P, Tai J, eds. Developing Evaluative Judgement in Higher education. New York: Routledge Taylor \& Francis group; 2018:23-33.

20. Jackel B, Pearce J, Radloff A, Edwards D. Assessment and Feedback in Higher Education: A Review of Literature for the Higher Education Academy. ACEReSearch Repository of research-based knowledge to improve learning. Higher Education Research Web site. https://research.acer.edu.au/higher_education/53/. Published 2017. Accessed January 2018, 2018.

21. Van der Kleij FM, Adie LE, Cumming JJ. A meta-review of the student role in feedback. International Journal of Educational Research. 2019;98:303-323.

22. Moher D, Liberati A, Tetzlaff J, Altman DG. Preferred reporting items for systematic reviews and meta-analyses: the PRISMA statement. Ann Intern Med. 2009;151(4):264-269.

23. PRISMA. http://www.prisma-statement.org/. Accessed February 2017, 2017. 
24. Higgins JPT, Green S, Wiley B, Cochrane C. Cochrane handbook for systematic reviews of interventions. Chichester, England: Wiley-Blackwell; 2008.

25. Kim SY, Park JE, Lee YJ, et al. Testing a tool for assessing the risk of bias for nonrandomized studies showed moderate reliability and promising validity. J Clin Epidemiol. 2013;66(4):408-414.

26. Nordheim LV, Gundersen MW, Espehaug B, Guttersrud $\varnothing$, Flottorp S. Effects of school-based educational interventions for enhancing adolescents abilities in critical appraisal of health claims: a systematic review. 2016;11(8):e0161485.

27. Feil PH, Reed T. The effect of knowledge of the desired outcome on dental motor performance. J Dent Educ. 1988;52(4):198-201.

28. Shah DY, Dadpe AM, Kalra DD, Garcha VP. Videotaped feedback method to enhance learning in preclinical operative dentistry: An experimental study. J Dent Educ. 2015;79(12):1461-1466.

29. Knight GW, Guenzel P. Discrimination training and formative evaluation for remediation in basic waxing skills. J Dent Educ. 1990;54(3):194-198.

30. Huth KC, Baumann M, Kollmuss M, Hickel R, Fischer MR, Paschos E. Assessment of practical tasks in the Phantom course of Conservative Dentistry by pre-defined criteria: a comparison between self-assessment by students and assessment by instructors. Eur J Dent Educ. 2017;21(1):37-45.

31. Manogue M, Brown G, Nattress B, Fox K. Improving student learning in root canal treatment using self-assessment. Int Endod J. 1999;32(5):397-405.

32. Geissler P. Student self-assessment in dental technology. J Dent Educ. 1973;37(9):1921.

33. Curtis DA, Lind SL, Dellinges M, Setia G, Finzen FC. Dental students' self-assessment of preclinical examinations. J Dent Educ. 2008;72(3):265-277.

34. Chambers DW, LaBarre EE. The effects of student self-assessment on learning in removable prosthodontics laboratory. J Dent Educ. 2014;78(5):668-680.

35. Tuncer D, Arhun N, Yamanel K, Celik C, Dayangac B. Dental Students' Ability to Assess Their Performance in a Preclinical Restorative Course: Comparison of Students' and Faculty Members' Assessments. J Dent Educ. 2015;79(6):658-664.

36. Knight GW, Guenzel P, Fitzgerald M. Teaching recognition skills to improve products. J Dent Educ. 1990;54(12):739-742. 
37. De Peralta TL, Ramaswamy V, Karl E, Van Tubergen E, McLean ME, Fitzgerald M. Caries removal by first-year dental students: A multisource competency assessment strategy for reflective practice. J Dent Educ. 2017;81(1):87-95.

38. Radjaeipour G, Chambers DW, Geissberger M. Can performance on summative evaluation of wax-added dental anatomy projects be better predicted from the combination of supervised and unsupervised practice than from supervised practice alone? Eur J Dent Educ. 2016;20(4):237-240.

39. Reed T, Feil P, Greer D. The reliability and agreement of subtask assessments. J Dent Educ. 1988;52(10):554-557.

40. Chambers DW, LaBarre EE. Why professional judgment is better than objective description in dental faculty evaluations of student performance. J Dent Educ. 2014;78(5):681-693.

41. Barnett AG, van der Pols JC, Dobson AJ. Regression to the mean: what it is and how to deal with it. Int J Epidemiol. 2004;34(1):215-220.

42. Koletsi D, Madahar A, Fleming P, Pandis N. Statistical testing against baseline was common in dental research. J Clin Epidemiol. 2015;68(7):776-781.

43. Bridges SM, Wyatt-Smith CM, Botelho MG. Clinical Assessment Judgements and 'Connoisseurship': Surfacing Curriculum-Wide Standards Through Transdisciplinary Dialogue. In: Carless D, Bridges SM, Chan CKY, Glofcheski R, eds. Scaling up Assessment for Learning in Higher Education. Vol 5.2017:81-98.

44. Panadero $E$, Jonsson A. The use of scoring rubrics for formative assessment purposes revisited: A review. Educ Res Rev. 2013;9:129-144.

45. Joughin G. Assessment, learning and judgement in higher education: A critical review. In: Assessment, learning and judgement in higher education. Springer; 2009:1-15.

46. O'Donovan B, Rust C, Price M. A scholarly approach to solving the feedback dilemma in practice. Assess Eval High Edu. 2016;41(6):938-949.

47. Boud D, Ajjawi R, Dawson P, Tai J. Developing evaluative judgement in higher education: Assessment for knowing and producing quality work. Routledge; 2018.

48. Bloxham S, Boyd P, Orr S. Mark my words: the role of assessment criteria in UK higher education grading practices. Stud High Educ. 2011;36(6):655-670. 
49. Orsmond P, Merry S, Reiling K. The Use of Exemplars and Formative Feedback when Using Student Derived Marking Criteria in Peer and Self-assessment. Assess Eval High Edu. 2002;27(4):309-323.

50. Sadler DR. Specifying and Promulgating Achievement Standards. Oxford Rev Educ. 1987;13(2):191-209.

51. To J, Carless D. Making productive use of exemplars: Peer discussion and teacher guidance for positive transfer of strategies. J Furth High Educ. 2016;40(6):746-764.

52. Hendry GD, White P, Herbert C. Providing exemplar-based 'feedforward' before an assessment: The role of teacher explanation. Act Learn High Educ. 2016;17(2):99109.

53. Handley K, Williams L. From copying to learning: using exemplars to engage students with assessment criteria and feedback. Assess Eval High Edu. 2011;36(1):95-108.

54. Carless D, Chan KKH. Managing dialogic use of exemplars. Assess Eval High Edu. 2017;42(6):930-941. 
Table 1: Search strategy details

Database: PubMed

\begin{tabular}{llr} 
Search number & Query & \multicolumn{2}{c}{ Results } \\
\hline$\# 14$ & $(\# 9)$ AND (\#8) & 691 \\
$\# 13$ & $(\# 9)$ AND (\#7) & 397 \\
$\# 11$ & $(\# 9)$ AND (\#6) & 752 \\
$\# 10$ & $((\# 9)$ AND (\#8)) OR (\#7) & 688,037 \\
$\# 9$ & $(((\# 1)$ OR (\#2)) AND (\#3)) AND (\#5) & 1,149 \\
$\# 8$ & teaching & $1,401,462$ \\
$\# 7$ & learning & 687,686 \\
$\# 6$ & education & $1,728,872$ \\
$\# 5$ & dentistry & 595,499 \\
$\# 4$ & dental & 576,057 \\
$\# 3$ & feedback & 157,489 \\
$\# 2$ & evaluation & $3,864,031$ \\
$\# 1$ & assessment & $3,270,975$ \\
\hline
\end{tabular}


Table 2: Design features of selected studies

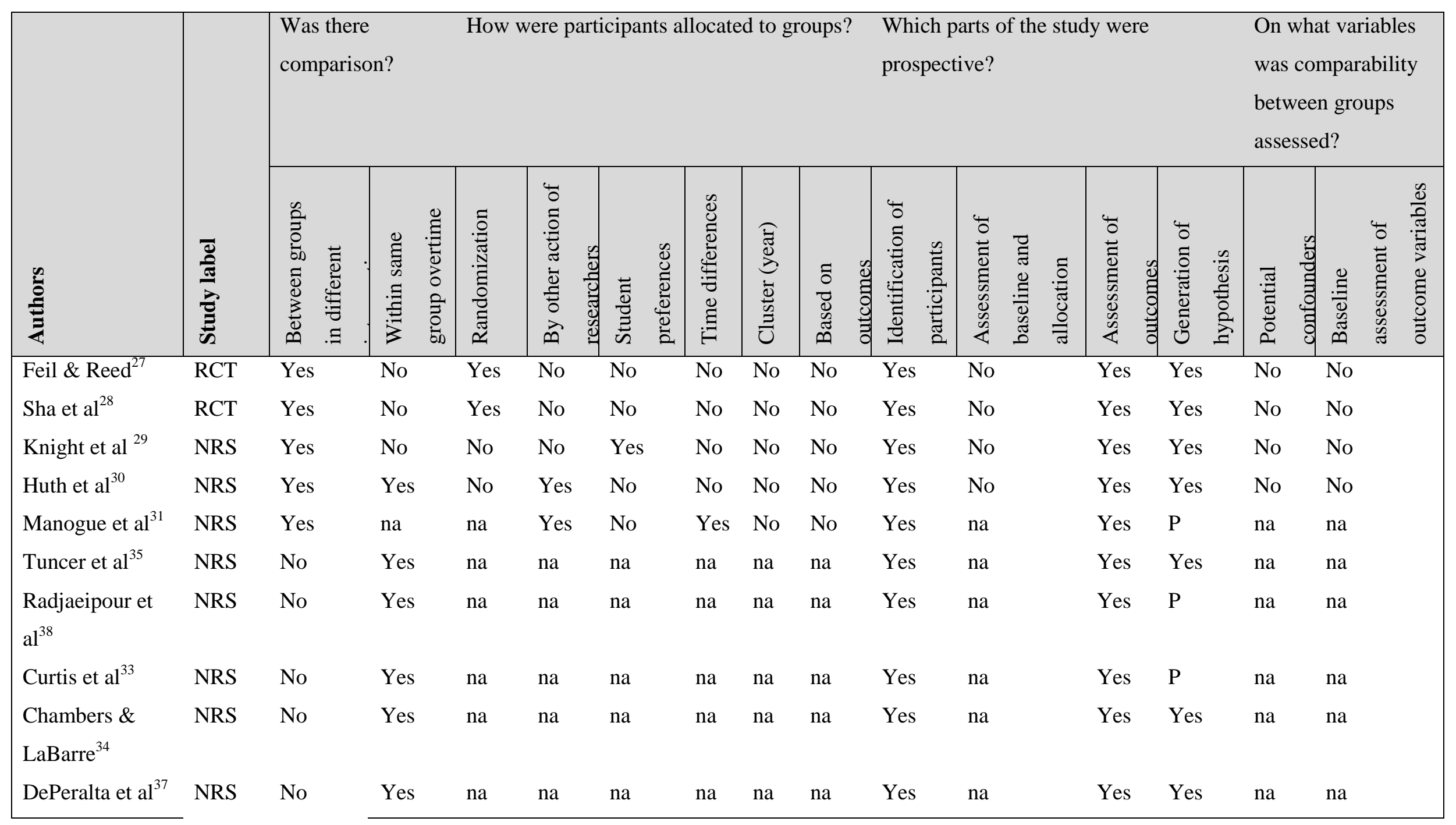

This article is protected by copyright. All rights reserved 


\begin{tabular}{|c|c|c|c|c|c|c|c|c|c|c|c|c|c|c|c|}
\hline Knight et al ${ }^{36}$ & NRS & No & Yes & na & na & na & na & na & na & Yes & na & Yes & Yes & na & na \\
\hline Geissler $^{32}$ & NRS & No & Yes & na & na & na & na & na & na & Yes & na & Yes & $\mathrm{P}$ & na & na \\
\hline
\end{tabular}

\begin{tabular}{|c|c|c|c|c|c|c|}
\hline First author & $\begin{array}{l}\text { Selection of } \\
\text { participants }\end{array}$ & $\begin{array}{l}\text { Confounding } \\
\text { variables }\end{array}$ & $\begin{array}{l}\text { Measurement } \\
\text { exposure }\end{array}$ & $\begin{array}{l}\text { Blinding of outcome } \\
\text { assessment }\end{array}$ & $\begin{array}{l}\text { Incomplete } \\
\text { outcome data }\end{array}$ & $\begin{array}{l}\text { Selective outcome } \\
\text { reporting }\end{array}$ \\
\hline Knight $^{29}$ & Low & Low & Low & Low & Low & Low \\
\hline Huth $^{30}$ & Low & Low & Low & Unclear & Low & Low \\
\hline Manogue $^{31}$ & High & High & Unclear & Unclear & Low & Low \\
\hline Tuncer $^{35}$ & Low & Low & Unclear & Unclear & Low & Low \\
\hline Radjaeipour $^{38}$ & Low & Unclear & Low & Low & Low & Low \\
\hline Curtis $^{33}$ & Low & High & Low & Low & Low & Low \\
\hline Chambers $^{34}$ & Low & Low & Low & Unclear & Low & Low \\
\hline De Peralta $^{37}$ & Low & Unclear & Low & Unclear & Low & Low \\
\hline Knight $^{36}$ & Low & Low & Low & Low & Low & Low \\
\hline
\end{tabular}

This article is protected by copyright. All rights reserved 


\begin{tabular}{|l|l|l|l|l|l|l|}
\hline Geissler $^{32}$ & Low & High & Low & Unclear & Low & Low \\
\hline
\end{tabular}

Table 4: Feedback intervention components ${ }^{8}$

This article is protected by copyright. All rights reserved 


\begin{tabular}{|c|c|c|c|c|c|c|c|c|c|c|c|c|c|c|}
\hline \multirow{2}{*}{$\begin{array}{l}\text { Intervention } \\
\text { cluster }\end{array}$} & \multirow[t]{2}{*}{ Intervention component } & \multicolumn{12}{|c|}{ Selected studies (first author initial) } & \multirow[b]{2}{*}{$\%$} \\
\hline & & $\mathrm{G}^{32}$ & $\mathrm{M}^{31}$ & $\mathrm{C}^{33}$ & $\mathrm{Ch}^{34}$ & $\mathrm{R}^{38}$ & $\mathrm{~T}^{35}$ & $\mathrm{~F}^{27}$ & $\mathrm{~K}^{29} \& \mathrm{~K}^{36}$ & $\mathrm{H}^{30}$ & $\mathrm{~S}^{28}$ & $\begin{array}{l}\text { DP } \\
37\end{array}$ & Total & \\
\hline \multirow{4}{*}{$\begin{array}{l}\text { 1.Internalising } \\
\text { and applying } \\
\text { standards }\end{array}$} & Self-assessment & $\mathrm{x}$ & $\mathrm{x}$ & $\mathrm{x}$ & $\mathrm{x}$ & $\mathrm{x}$ & $\mathrm{x}$ & $\mathrm{x}$ & $\mathrm{x}$ & $\mathrm{x}$ & $\mathrm{x}$ & $\mathrm{x}$ & 12 & 100 \\
\hline & Peer assessment & & & & $\mathrm{x}$ & & $\mathrm{X}$ & & $\mathrm{x}$ & & & $\mathrm{x}$ & 4 & 33 \\
\hline & Engaging with grading criteria & $\mathrm{X}$ & $\mathrm{X}$ & $\mathrm{x}$ & $\mathrm{X}$ & $\mathrm{X}$ & $\mathrm{X}$ & $\mathrm{x}$ & $\mathrm{x}$ & $\mathrm{x}$ & $\mathrm{X}$ & $\mathrm{X}$ & 12 & 100 \\
\hline & Dialogue and discussion & $\mathrm{x}$ & $\mathrm{x}$ & & & $\mathrm{x}$ & & & $\mathrm{x}$ & $\mathrm{x}$ & $\mathrm{x}$ & $\mathrm{x}$ & 7 & 58 \\
\hline 2.Sustainable & Action planning & & & & & & & & $\mathrm{x}$ & & & $\mathrm{x}$ & 2 & 16 \\
\hline monitoring & Portfolio & & & & & & & & & & & & & \\
\hline 3.Collective & Feedback workshop & & & & & & $\mathrm{X}$ & $\mathrm{X}$ & $\mathrm{X}$ & & & & 4 & 33 \\
\hline provision of & Feedback resources & & $\mathrm{x}$ & & & & & & & & & & 1 & 8 \\
\hline training & Exemplar assignments & & & & & & & $\mathrm{x}$ & $\mathrm{x}$ & $\mathrm{x}$ & $\mathrm{X}$ & & 4 & 33 \\
\hline 4.Manner of & Formative assessment & $\mathrm{X}$ & $\mathrm{X}$ & $\mathrm{x}$ & $\mathrm{X}$ & $\mathrm{X}$ & $\mathrm{X}$ & $\mathrm{X}$ & $\mathrm{X}$ & $\mathrm{x}$ & $\mathrm{X}$ & $\mathrm{X}$ & 12 & 100 \\
\hline feedback & Feedback without grade & & $\mathrm{X}$ & & & & & & & & & & 1 & 8 \\
\hline \multirow[t]{3}{*}{ delivery } & Tailored feedback & & & & & & & $\mathrm{x}$ & & & & & 1 & 8 \\
\hline & Presentation of feedback & & & & & & & & & & & & & \\
\hline & Technology & & & & & & & & & & $\mathrm{x}$ & & 1 & 8 \\
\hline
\end{tabular}

$\mathrm{X}$ : Indicates the intervention component is present in the study

This article is protected by copyright. All rights reserved 
Table 5 Characteristics of selected studies

\begin{tabular}{|c|c|c|c|c|c|c|}
\hline Geissler $1973^{32}$ & $\begin{array}{l}\text { United } \\
\text { Kingdom }\end{array}$ & $\begin{array}{l}\text { n: } 9 \text { Age: not } \\
\text { reported Gender: } \\
\text { not reported }\end{array}$ & $\begin{array}{l}\text { Faculty } \\
\text { instructors }\end{array}$ & $\begin{array}{l}\text { Intervention: Student self- } \\
\text { assessment and instructor- } \\
\text { student discussions. } \\
\text { Comparison: Students } \\
\text { progress measured in three } \\
\text { different occasions. }\end{array}$ & 4 months & $\begin{array}{l}\text { Lectures, } \\
\text { demonstrations } \\
\text { and practical } \\
\text { activities. }\end{array}$ \\
\hline $\begin{array}{l}\text { Manogue et al } \\
1999^{31}\end{array}$ & $\begin{array}{l}\text { United } \\
\text { Kingdom }\end{array}$ & $\begin{array}{l}\text { n: } 98 \text { Age: not } \\
\text { reported Gender: } \\
\text { not reported }\end{array}$ & $\begin{array}{l}\text { Faculty } \\
\text { instructors and } \\
\text { lecturer. }\end{array}$ & $\begin{array}{l}\text { Intervention: } 3 \text { lectures } \\
8 \text { hrs tutorials, } 5 \mathrm{~h} \\
\text { independent study time } 21 \\
\mathrm{~h} \text { practical classes with } \\
\text { self-assessment } 5 \mathrm{~h} \\
\text { practical test with self- } \\
\text { assessment. Comparison: }\end{array}$ & $\begin{array}{l}\text { Intervention: } 34 \\
\text { contact hrs. } \\
\text { Original: } 36 \\
\text { contact hours }\end{array}$ & $\begin{array}{l}\text { Lectures, tutorials } \\
\text { and practical } \\
\text { activities. }\end{array}$ \\
\hline
\end{tabular}

This article is protected by copyright. All rights reserved 


\begin{tabular}{|c|c|c|c|c|c|c|}
\hline & & & & $\begin{array}{l}8 \text { lectures, } 39 \text { h practical } \\
\text { work, } 3 \text { h operative test. }\end{array}$ & & \\
\hline $\begin{array}{l}\text { Curtis et al } 2007 \\
33\end{array}$ & United States & $\begin{array}{l}\text { n: } 77 \text { Age: Not } \\
\text { reported Gender: } \\
48 \text { females, } 29 \\
\text { males. }\end{array}$ & $\begin{array}{l}\text { Faculty } \\
\text { instructors }\end{array}$ & $\begin{array}{l}\text { Intervention: } \\
\text { Student self- and faculty } \\
\text { assessment of } \\
\text { prosthodontic exam. } \\
\text { Comparison: Differences } \\
\text { between first and second } \\
\text { attempt on students' self } \\
\text { and tutors' assessments }\end{array}$ & $\begin{array}{l}\text { Two identical } \\
\text { three hours } \\
\text { examination } \\
\text { spaced by } 5 \\
\text { weeks }\end{array}$ & $\begin{array}{l}\text { Instruction and a } \\
\text { review of the } \\
\text { evaluation format } \\
\text { before first } \\
\text { examination } \\
\text { attempt. }\end{array}$ \\
\hline $\begin{array}{l}\text { Chambers and } \\
\text { LaBarre } 2013^{34}\end{array}$ & United States & $\begin{array}{l}\text { n: } 157,135 \text { DDS } \\
\text { and } 27 \\
\text { international } \\
\text { students. Age: } \\
\text { Not reported } \\
\text { Gender: not } \\
\text { reported }\end{array}$ & $\begin{array}{l}\text { Faculty } \\
\text { instructors }\end{array}$ & $\begin{array}{l}\text { Intervention: Two } \\
\text { practical projects with } \\
\text { students' self-assessment } \\
\text { and tutors' assessments. } \\
\text { Comparison: Student and } \\
\text { faculty scores in first and } \\
\text { second attempt }\end{array}$ & 6 months & $\begin{array}{l}\text { Practical projects: } \\
\text { Self, peer and } \\
\text { faculty } \\
\text { assessments of } \\
\text { each project }\end{array}$ \\
\hline
\end{tabular}

This article is protected by copyright. All rights reserved 


\begin{tabular}{|c|c|c|c|c|c|c|}
\hline $\begin{array}{l}\text { Radjaeipour et al } \\
2015^{38}\end{array}$ & United States & $\begin{array}{l}\text { n: } 562 \text { (four } \\
\text { consecutive } \\
\text { classes) Age: } \\
\text { majority in their } \\
\text { 20s Gender: ratio } \\
\text { male female } \\
+15 \%\end{array}$ & $\begin{array}{l}8 \text { to } 12 \text { faculty } \\
\text { instructors }\end{array}$ & $\begin{array}{l}\text { Intervention: Wax up } \\
\text { project at home without } \\
\text { assistance, wax up project } \\
\text { with assistance in the } \\
\text { laboratory, wax up } \\
\text { practical projects (exams). } \\
\text { Comparison: score of } \\
\text { different projects. }\end{array}$ & $\begin{array}{l}3 \text { or } 4 \text { weeks for } \\
\text { home project } \\
\text { and laboratory } \\
\text { project. A total } \\
\text { of } 30 \text { session } \\
\text { preceded by } 1 \mathrm{hr} \\
\text { lecture. } 2.5 \text { to } 3 \\
\text { hrs for practical } \\
\text { projects. } \\
\text { Data collected } \\
\text { for four years. } \\
\text { A full term, no } \\
\text { details given }\end{array}$ & $\begin{array}{l}\text { Lectures and } \\
\text { laboratory } \\
\text { sessions. } \\
\text { Community } \\
\text { driven e-learning } \\
\text { platform, books, } \\
\text { notes waxing } \\
\text { schemes, grading } \\
\text { forms. }\end{array}$ \\
\hline
\end{tabular}

This article is protected by copyright. All rights reserved 


\begin{tabular}{|c|c|c|c|c|c|c|}
\hline $\begin{array}{l}\text { Feil and Reed } \\
1988^{27}\end{array}$ & United States & $\begin{array}{l}\text { n: } 99 \text { Age: not } \\
\text { reported Gender: } \\
\text { not reported }\end{array}$ & $\begin{array}{l}\text { Faculty } \\
\text { instructors }\end{array}$ & $\begin{array}{l}\text { Intervention: Students } \\
\text { exposed different quality } \\
\text { preparations. Instructor } \\
\text { centred + student centred. } \\
\text { Comparison: Instructor } \\
\text { centred }\end{array}$ & $\begin{array}{l}\text { Two sessions, } \\
\text { experimental } \\
\text { group } 45 \\
\text { minutes more } \\
\text { than the control. }\end{array}$ & $\begin{array}{l}\text { Student and } \\
\text { teacher centred in } \\
\text { the intervention, } \\
\text { teacher centred } \\
\text { instruction in the } \\
\text { control }\end{array}$ \\
\hline $\begin{array}{l}\text { Knight et al } \\
1990^{36}\end{array}$ & United States & $\begin{array}{l}\text { n: } 86 \text { Age: not } \\
\text { reported Gender: } \\
\text { not reported }\end{array}$ & $\begin{array}{l}\text { Faculty } \\
\text { instructors }\end{array}$ & $\begin{array}{l}\text { Intervention: Recognition } \\
\text { of ideal and variants using } \\
\text { criteria with pictures and } \\
\text { real models. } \\
\text { Demonstration of } \\
\text { evaluation and group } \\
\text { discussion, production } \\
\text { training, self- evaluation, } \\
\text { discussion with peers, } \\
\text { formative assessment. } \\
\text { Comparison: Student and } \\
\text { faculty scores overtime. }\end{array}$ & 16 hours & $\begin{array}{l}\text { Student centred, } \\
\text { teacher } \\
\text { supervision, } \\
\text { television } \\
\text { presentations }\end{array}$ \\
\hline
\end{tabular}

This article is protected by copyright. All rights reserved 


\begin{tabular}{|c|c|c|c|c|c|c|}
\hline $\begin{array}{l}\text { Knight et al } \\
1990^{29}\end{array}$ & United States & $\begin{array}{l}\text { n: } 13 \text { Age: not } \\
\text { reported Gender: } \\
\text { not reported }\end{array}$ & $\begin{array}{l}\text { Faculty } \\
\text { instructors }\end{array}$ & $\begin{array}{l}\text { Intervention: Recognition } \\
\text { of ideal and variants using } \\
\text { criteria with pictures and } \\
\text { real models. } \\
\text { Demonstration of } \\
\text { evaluation technique and } \\
\text { group discussion, } \\
\text { production training, self- } \\
\text { evaluation, discussion with } \\
\text { peers, formative } \\
\text { assessment. } \\
\text { Comparison: Received } \\
\text { verbal feedback with } \\
\text { models on seven waxing } \\
\text { projects over } 13 \text { hrs }\end{array}$ & 13 hours & Not provided \\
\hline
\end{tabular}

This article is protected by copyright. All rights reserved 


\begin{tabular}{|c|c|c|c|c|c|c|}
\hline Huth et al $2017^{30}$ & Germany & $\begin{array}{l}\text { n: } 42 . \text { Gender: } 17 \\
\text { male } 25 \text { female } \\
\text { Age: not provided }\end{array}$ & $\begin{array}{l}\text { Faculty } \\
\text { instructors }\end{array}$ & $\begin{array}{l}\text { Intervention: Tree training } \\
\text { intensities: I no additional } \\
\text { assessment of model, not } \\
\text { discussion with peers, no } \\
\text { feedback by instructor II } \\
\text { one assessment of a test } \\
\text { model, no discussion with } \\
\text { peers, feedback by } \\
\text { instructor III Two } \\
\text { assessments of test } \\
\text { models, discussion with } \\
\text { peers, feedback by } \\
\text { instructor. } \\
\text { Comparison: Between } \\
\text { interventions and Student } \\
\text { and faculty scores } \\
\text { overtime. }\end{array}$ & One term & $\begin{array}{l}\text { Lectures on } \\
\text { procedures. } \\
\text { Interactive use of } \\
\text { marking criteria } \\
\text { on examples of } \\
\text { different quality. }\end{array}$ \\
\hline $\begin{array}{l}\text { Shah et al } 2015^{28} \\
\text { Randomised } \\
\text { controlled trial }\end{array}$ & India & $\begin{array}{l}\text { n: } 60 \text { Age: } \\
\text { average } 19 \text { years } \\
\text { old Gender: } \\
\text { female } 47 \text { male }\end{array}$ & $\begin{array}{l}\text { Faculty } \\
\text { instructors }\end{array}$ & $\begin{array}{l}\text { Intervention: Feedback } \\
\text { session aided with } \\
\text { videotape (interactive } \\
\text { feedback session) Student }\end{array}$ & $1 \mathrm{hr} 15$ minutes & $\begin{array}{l}\text { Video } \\
\text { demonstration }\end{array}$ \\
\hline
\end{tabular}

This article is protected by copyright. All rights reserved 


\begin{tabular}{|c|c|c|c|c|c|c|}
\hline $\begin{array}{l}\text { De Peralta et al } \\
2017^{37}\end{array}$ & United states & $\begin{array}{l}\text { n: } 104 \text { Age: not } \\
\text { reported Gender: } \\
\text { not reported }\end{array}$ & $\begin{array}{l}\text { Faculty } \\
\text { instructors } \\
\text { 8:1 student: } \\
\text { faculty ratio }\end{array}$ & $\begin{array}{l}\text { and faculty scores } \\
\text { Comparison: control group } \\
\text { without videotaped } \\
\text { feedback session Student } \\
\text { and faculty scores } \\
\text { Intervention: Multisource } \\
\text { assessment and reflective } \\
\text { practice. Comparison: } \\
\text { Student and faculty scores } \\
\text { overtime. }\end{array}$ & Two weeks & $\begin{array}{l}\text { Video instruction } \\
\text { small groups }\end{array}$ \\
\hline
\end{tabular}

This article is protected by copyright. All rights reserved 
Table 5 cont: Characteristics of selected studies

\begin{tabular}{|c|c|c|c|c|c|}
\hline $\begin{array}{l}\text { First author and } \\
\text { year of publication }\end{array}$ & Discipline & Task & Measured Outcome & $\begin{array}{c}\text { Type of } \\
\text { measurement }\end{array}$ & $\begin{array}{l}\text { Graders reliability } \\
\text { strategies }\end{array}$ \\
\hline Geissler $1973^{32}$ & Prosthodontics & $\begin{array}{l}\text { Construction of } \\
\text { complete and } \\
\text { removable denture }\end{array}$ & $\begin{array}{l}\text { Correlation between } \\
\text { students and expert } \\
\text { marks reported in \% }\end{array}$ & $\begin{array}{l}\text { For each criterion } \\
\text { (12): Good, } \\
\text { satisfactory and poor }\end{array}$ & Not reported \\
\hline $\begin{array}{l}\text { Manogue et al } 1999 \\
31\end{array}$ & $\begin{array}{l}\text { Endodontics and } \\
\text { fixed prosthodontics }\end{array}$ & $\begin{array}{l}\text { Endodontics and } \\
\text { crown preparation }\end{array}$ & $\begin{array}{l}\text { Students self- } \\
\text { reported confidence } \\
\text { questionnaire. } \\
\text { Problem solving } \\
\text { ability }\end{array}$ & $\begin{array}{l}\text { Questionnaire using } \\
\text { a } 1 \text { to } 5 \text { (Strongly } \\
\text { disagree to strongly } \\
\text { agree) scale. } \\
\text { Tutors comments. }\end{array}$ & Not provided \\
\hline Curtis et al $2007^{33}$ & Prosthodontics & $\begin{array}{l}\text { Setting maxillary } \\
\text { denture teeth against } \\
\text { standardised lower } \\
\text { cast. } \\
\text { Summative exam. }\end{array}$ & $\begin{array}{l}\text { Correlation between } \\
\text { students' self- } \\
\text { assessment scores } \\
\text { and teachers scores. } \\
\text { Validated self- } \\
\text { assessment: adjusted } \\
\text { score for agreement } \\
\text { between assessor and }\end{array}$ & $\begin{array}{l}\text { Evaluation criteria: } \\
\text { denture tooth } \\
\text { position, arch form, } \\
\text { midline positioning } \\
\text { of teeth, contact in } \\
\text { centric relation, and } \\
\text { functional measures } \\
\text { in working, }\end{array}$ & $\begin{array}{l}\text { Reliability: } \\
\text { Single evaluator } \\
\text { evaluated } 10 \\
\text { dentures twice } r=.77\end{array}$ \\
\hline
\end{tabular}

This article is protected by copyright. All rights reserved 


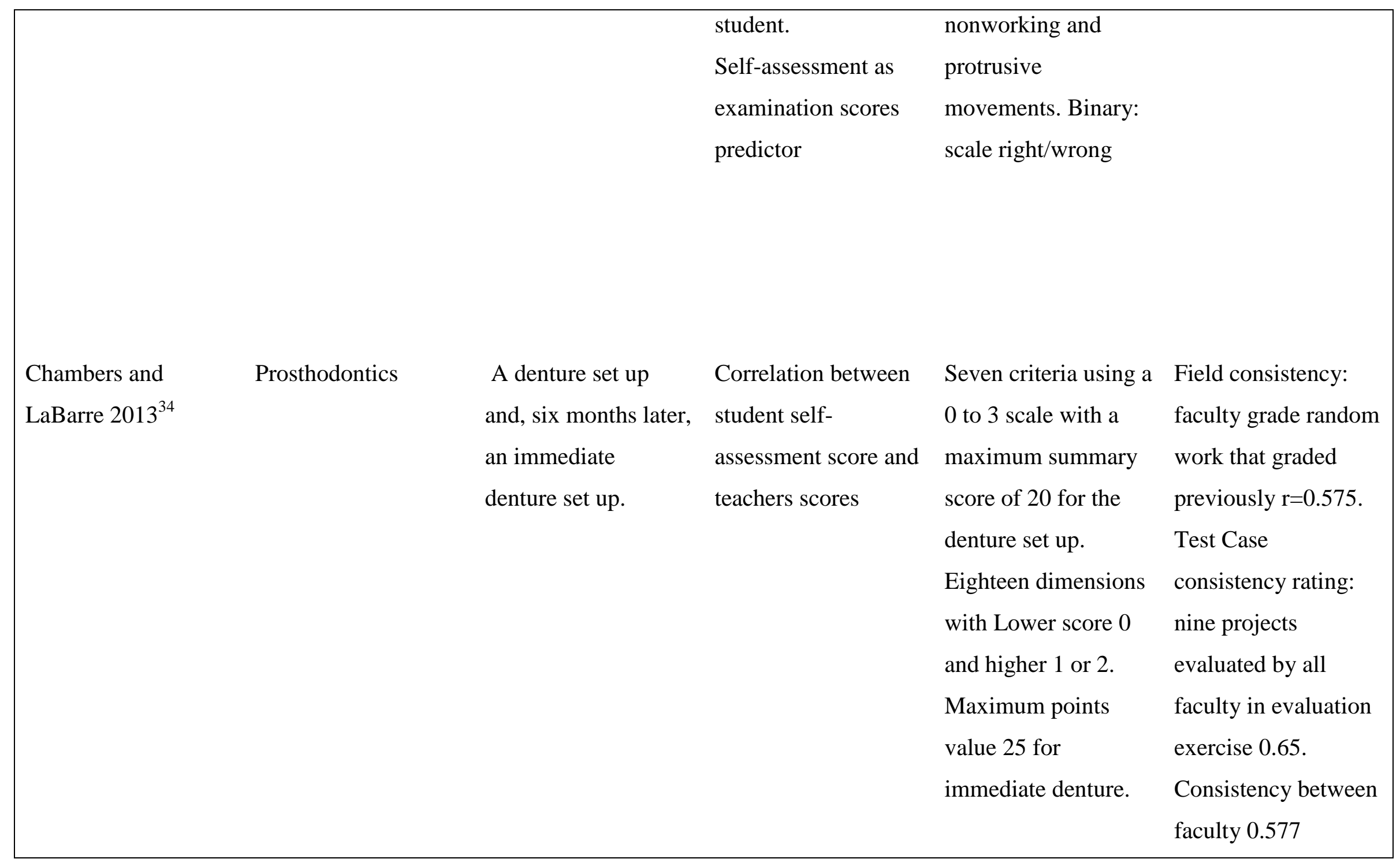

This article is protected by copyright. All rights reserved 


\begin{tabular}{|c|c|c|c|c|c|}
\hline $\begin{array}{l}\text { Radjaeipour et al } \\
2015^{38}\end{array}$ & Dental anatomy & $\begin{array}{l}\text { Home project wax } \\
\text { ups (7), laboratory } \\
\text { projects (6), practical } \\
\text { projects (4) }\end{array}$ & $\begin{array}{l}\text { Products scores } \\
\text { reported in } \\
\text { percentages. } \\
\text { Correlation between } \\
\text { home projects, } \\
\text { laboratory projects } \\
\text { and practical projects }\end{array}$ & $\begin{array}{l}\text { Standardised scoring } \\
\text { rubrics. }\end{array}$ & $\begin{array}{l}\text { Marking Protocol } \\
\text { described. IRR or } \\
\text { other measures of } \\
\text { consistency not } \\
\text { provided. }\end{array}$ \\
\hline Tuncer et al $2015^{35}$ & Operative Dentistry & $\begin{array}{l}\text { 2nd year: Amalgam. } \\
\text { 3rd year: Amalgam } \\
\text { and composite. }\end{array}$ & $\begin{array}{l}\text { Product scores, self } \\
\text { and faculty graded } \\
\text { reported in means }\end{array}$ & $\begin{array}{l}\text { Criteria not } \\
\text { specified, out of } 100 \\
\text { points. }\end{array}$ & $\begin{array}{l}\text { Experienced } \\
\text { assessors }(n=3) \text {. } \\
\text { Level of agreement } \\
\text { not reported }\end{array}$ \\
\hline $\begin{array}{l}\text { Feil and Reed } 1988 \\
27\end{array}$ & Operative Dentistry & $\begin{array}{l}\text { Class I and II } \\
\text { preparations for } \\
\text { amalgam }\end{array}$ & $\begin{array}{l}\text { Agreement between } \\
\text { students and faculty } \\
\text { grades. Students } \\
\text { performance. }\end{array}$ & Grades A, B, C and F & $\begin{array}{l}\text { Intra rater reliability } \\
\text { class } \mathrm{I}=.79 \text { class } \mathrm{II}= \\
.71\end{array}$ \\
\hline Knight et al $1990^{36}$ & Operative Dentistry & Class I for amalgam & $\begin{array}{l}\text { Recognition skills } \\
\text { (agreement with } \\
\text { faculty pre and post } \\
\text { products) and } \\
\text { production skills }\end{array}$ & $\begin{array}{l}\text { Recognition skills: } 1 \\
\text { to } 10 \text { points. } \\
\text { Production skills: } 5 \\
\text { points referenced } \\
\text { scale ( } 8 \text { criteria). }\end{array}$ & $\begin{array}{l}\text { Faculty (2) } \\
\text { agreement } 82.3 \% \\
\text { Kappa=.75 }\end{array}$ \\
\hline
\end{tabular}

This article is protected by copyright. All rights reserved 


\begin{tabular}{|c|c|c|c|c|c|}
\hline Knight et al $1990^{29}$ & Operative Dentistry & $\begin{array}{l}\text { Waxing life size } \\
\text { plastic teeth }\end{array}$ & $\begin{array}{l}\text { Recognition skills } \\
\text { (agreement with } \\
\text { faculty pre and post } \\
\text { products) and } \\
\text { production skills }\end{array}$ & Marking criteria & $\begin{array}{l}\text { Perfect agreement } \\
\text { evaluators } 82.3 \% \\
\text { Kappa=0.65 }\end{array}$ \\
\hline Huth et al $2015^{30}$. & Restorative dentistry & $\begin{array}{l}\text { Preparation for } \\
\text { composite, } \\
\text { composite filling, } \\
\text { preparation for gold, } \\
\text { preparation for } \\
\text { ceramic, trepanation, } \\
\text { root canal filling }\end{array}$ & $\begin{array}{l}\text { Students ratings for } \\
\text { each task and } \\
\text { training intensity. } \\
\text { Agreement } \\
\text { student/instructor } \\
\text { rates. Attitudes } \\
\text { towards intervention. }\end{array}$ & $\begin{array}{l}\text { Standardised } \\
\text { assessment criteria. } \\
\text { Likert } 4 \text { points scale }\end{array}$ & $\begin{array}{l}\text { ICC: } 0.944 \text { for } \\
\text { instructors } 0.949 \text { for } \\
\text { students. }\end{array}$ \\
\hline Shah et al $2015^{28}$ & Operative Dentistry & $\begin{array}{l}\text { Class II preparation } \\
\text { for amalgam }\end{array}$ & $\begin{array}{l}\text { Mean grades self - } \\
\text { assessment and } \\
\text { tutors scores } \\
\text { Questionnaire } \\
\text { answers } \\
\text { (Intervention group } \\
\text { only) }\end{array}$ & $\begin{array}{l}\text { A scoring check list } \\
\text { (no details provided) } \\
5 \text { points Likert scale } \\
1 \text { strongly disagree to } \\
5 \text { strongly agree }\end{array}$ & Not reported \\
\hline
\end{tabular}

This article is protected by copyright. All rights reserved 


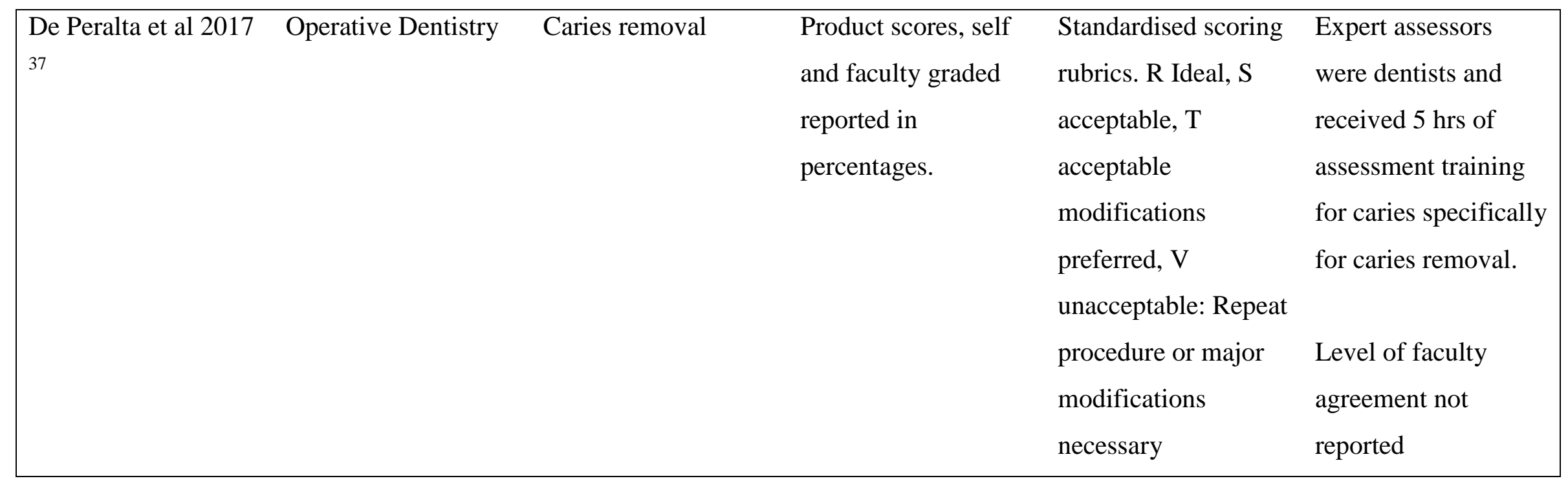

This article is protected by copyright. All rights reserved 
Figure legends

Figure 1: Systematic review flow chart of the literature search and selection process Figure 2: Risk of bias for Randomised Controlled Trials

Appendix 1: Descriptions of each intervention component type

Appendix 2: Risk of bias assessment in included non-randomised studies

Appendix 3: Excluded papers with reasons 


\section{Appendix 1: Descriptions of each intervention component type ${ }^{25}$}

\begin{tabular}{|c|c|c|}
\hline $\begin{array}{l}\text { Intervention } \\
\text { cluster }\end{array}$ & $\begin{array}{l}\text { Intervention } \\
\text { component }\end{array}$ & Description \\
\hline \multirow[t]{4}{*}{$\begin{array}{l}\text { Internalising } \\
\text { and applying } \\
\text { standards }\end{array}$} & $\begin{array}{l}\text { Peer } \\
\text { assessment }\end{array}$ & $\begin{array}{l}\text { Use of learners' own peers as a source of feedback and } \\
\text { assessment, and/or assessing the work of one's peers as a } \\
\text { means to engage with the expected standards }\end{array}$ \\
\hline & $\begin{array}{l}\text { Self- } \\
\text { assessment }\end{array}$ & $\begin{array}{l}\text { Requiring or encouraging learners to assess and prepare } \\
\text { feedback on their own work, as a means to engage with the } \\
\text { expected standards }\end{array}$ \\
\hline & $\begin{array}{l}\text { Engaging } \\
\text { with grading } \\
\text { criteria }\end{array}$ & $\begin{array}{l}\text { Providing structured procedures or resources to help } \\
\text { learners to understand grading criteria }\end{array}$ \\
\hline & $\begin{array}{l}\text { Dialogue and } \\
\text { discussion }\end{array}$ & $\begin{array}{l}\text { Requiring or encouraging learners to talk to their educators } \\
\text { and seek advice, either as a substitute for, or in addition to } \\
\text { receiving written feedback }\end{array}$ \\
\hline \multirow[t]{2}{*}{$\begin{array}{l}\text { Sustainable } \\
\text { monitoring }\end{array}$} & $\begin{array}{l}\text { Action } \\
\text { planning }\end{array}$ & $\begin{array}{l}\text { Forcing or encouraging learners to set themselves specific } \\
\text { goals on the basis of their feedback, and to make clear } \\
\text { action plans as means to work toward those goals }\end{array}$ \\
\hline & Portfolio & $\begin{array}{l}\text { Collecting learners' feedback together in one folder, or } \\
\text { online profile, so that many sets of feedback can be viewed } \\
\text { together and progress over time can be easily tracked }\end{array}$ \\
\hline \multirow[t]{3}{*}{$\begin{array}{l}\text { Collective } \\
\text { provision of } \\
\text { training }\end{array}$} & $\begin{array}{l}\text { Feedback } \\
\text { workshop }\end{array}$ & $\begin{array}{l}\text { Structured sessions in which advice and/or activities are } \\
\text { delivered to learners, intended to help them understand } \\
\text { how to engage with and use feedback }\end{array}$ \\
\hline & $\begin{array}{l}\text { Feedback } \\
\text { resources }\end{array}$ & $\begin{array}{l}\text { Providing guidance documents that learners can use } \\
\text { independently to improve their strategies for using } \\
\text { feedback }\end{array}$ \\
\hline & $\begin{array}{l}\text { Exemplar } \\
\text { assignments }\end{array}$ & $\begin{array}{l}\text { Providing learners with real and/or constructed examples } \\
\text { of previous assignments, as means to demonstrate optimal }\end{array}$ \\
\hline
\end{tabular}




\begin{tabular}{|c|c|c|}
\hline & & $\begin{array}{l}\text { and/or suboptimal ways of completing their own } \\
\text { assignment, and what makes these optimal or suboptimal }\end{array}$ \\
\hline \multirow[t]{5}{*}{$\begin{array}{l}\text { Manner of } \\
\text { feedback } \\
\text { deliver }\end{array}$} & $\begin{array}{l}\text { Formative } \\
\text { assessment/ } \\
\text { Resubmission }\end{array}$ & $\begin{array}{l}\text { Providing learners with opportunities to submit and } \\
\text { receive feedback on noncredited work (as contrasted with } \\
\text { credit-bearing summative assessment) and/or allowing } \\
\text { learners to revise and resubmit their work after receiving } \\
\text { summative feedback }\end{array}$ \\
\hline & $\begin{array}{l}\text { Feedback } \\
\text { without a } \\
\text { grade }\end{array}$ & $\begin{array}{l}\text { Withholding grades from learners until they have engaged } \\
\text { with the qualitative feedback }\end{array}$ \\
\hline & $\begin{array}{l}\text { Tailored } \\
\text { feedback }\end{array}$ & $\begin{array}{l}\text { Allowing learners to specify which aspects of their work } \\
\text { they want to receive feedback on, and then giving } \\
\text { feedback tailored specifically to their preferences }\end{array}$ \\
\hline & $\begin{array}{l}\text { Presentation } \\
\text { of feedback }\end{array}$ & $\begin{array}{l}\text { Changing the way in which feedback is presented to } \\
\text { learners, through manipulations of visual layout, } \\
\text { presentation medium, level of detail, and so forth }\end{array}$ \\
\hline & Technology & $\begin{array}{l}\text { Use of learning technologies to aid feedback use, including } \\
\text { virtual learning environments, audio/video, computer- } \\
\text { generated feedback, electronic voting systems, and Short } \\
\text { Messaging Systems }\end{array}$ \\
\hline Other & Other & $\begin{array}{l}\text { Feedback interventions that do not fit into the preceding } \\
\text { categories. }\end{array}$ \\
\hline
\end{tabular}

This article is protected by copyright. All rights reserved 
Appendix 2: Risk of bias assessment in included non-randomised studies ${ }^{1}$

\begin{tabular}{|c|c|c|}
\hline \multicolumn{3}{|l|}{ Knight et al 1990* (NRCT) } \\
\hline Entry & Risk & Description \\
\hline Selection of participants & Low & $\begin{array}{l}\text { Intervention and control are from the same } \\
\text { population of students and participated during the } \\
\text { same time. All students failed to demonstrate } \\
\text { mastery of waxing skills of the course before the } \\
\text { intervention. }\end{array}$ \\
\hline Confounding variables & Low & $\begin{array}{l}\text { Students previous skills is disclosed. Investigators } \\
\text { did not find significant differences between the two } \\
\text { groups on entry GPA and academic records . }\end{array}$ \\
\hline Measurement of exposure & Low & Data measured by trained experts \\
\hline $\begin{array}{l}\text { Blinding of outcome } \\
\text { assessment }\end{array}$ & Unclear & \\
\hline Incomplete outcome data & Low & There is not missing data \\
\hline Free of selective reporting & Low & $\begin{array}{l}\text { Data reported is consistent with objectives and } \\
\text { methods. The same data as a previous study is } \\
\text { reported }\end{array}$ \\
\hline
\end{tabular}

\section{Curtis et al (NRS)}

\begin{tabular}{|l|l|l|}
\hline Entry & Risk & Description \\
\hline Selection of participants & Low & $\begin{array}{l}\text { All participants are from the same population and } \\
\text { data were collected prospectively }\end{array}$ \\
\hline Confounding variables & High & $\begin{array}{l}\text { Confounding factors such students' general } \\
\text { capability are not reported }\end{array}$ \\
\hline Measurement of exposure & Low & Data obtained from trained grader (r=.77) \\
\hline assessment & Low & $\begin{array}{l}\text { "The faculty member who graded all maxillary } \\
\text { dentures was mask to students' identity" }\end{array}$ \\
\hline Incomplete outcome data & Low & $\begin{array}{l}\text { One participant who did not complete second part } \\
\text { of study was removed from study. }\end{array}$ \\
\hline Free of selective reporting & Low & Data reported are consistent with study objectives \\
\hline
\end{tabular}

\section{Chambers et al (NRS)}

This article is protected by copyright. All rights reserved 


\begin{tabular}{|l|l|l|}
\hline Entry & Risk & Description \\
\hline Selection of participants & Low & $\begin{array}{l}\text { All participants are from the same population and } \\
\text { data were collected prospectively }\end{array}$ \\
\hline Confounding variables & Low & $\begin{array}{l}\text { Confounding factor such students' general } \\
\text { capability in laboratory and clinics are controlled }\end{array}$ \\
\hline Measurement of exposure & Low & $\begin{array}{l}\text { Comprehensive graders calibration strategies } \\
\text { reported }\end{array}$ \\
\hline Blinding of outcome & Unclear & $\begin{array}{l}\text { Graders could not see students' self-assessment but } \\
\text { it is not clear if graders could identify participants }\end{array}$ \\
\hline Incomplete outcome data & Low & $\begin{array}{l}\text { Number of participants is available and baseline } \\
\text { data did not differ from second part of intervention }\end{array}$ \\
\hline Free of selective reporting & Low & Reported data align with objectives \\
\hline
\end{tabular}

\begin{tabular}{|c|c|c|}
\hline \multicolumn{3}{|l|}{ Manogue et al (NRS) } \\
\hline Entry & Risk & Description \\
\hline Selection of participants & High & $\begin{array}{l}\text { "Comparison of the attitudes of two successive } \\
\text { cohorts of students towards... was undertaken in } \\
\text { two successive years" First year was the control for } \\
\text { the intervention in second year }\end{array}$ \\
\hline Confounding variables & High & $\begin{array}{l}\text { Confounding factors such students' general } \\
\text { capability are not reported }\end{array}$ \\
\hline Measurement of exposure & Unclear & \\
\hline $\begin{array}{l}\text { Blinding of outcome } \\
\text { assessment }\end{array}$ & Unclear & \\
\hline Incomplete outcome data & Low & $10 \%$ of questionnaires were not returned \\
\hline Free of selective reporting & Low & Data reported are consistent with study objectives \\
\hline
\end{tabular}

\begin{tabular}{|l|l|l|}
\hline Huth (NRS) & Risk & Description \\
\hline Entry & Low & $\begin{array}{l}\text { Intervention and control are from the same } \\
\text { population of students and participated during the } \\
\text { same time. }\end{array}$ \\
\hline Selection of participants & & A three arms design provided a baseline of \\
\hline Confounding variables & Low & \\
\hline
\end{tabular}




\begin{tabular}{|l|l|l|}
\hline Measurement of exposure & Low & $\begin{array}{l}\text { 'students' ability } \\
\text { and graders calibration strategies reported }\end{array}$ \\
\hline Blinding of outcome \\
assessment & Unclear & $\begin{array}{l}\text { Graders were blinded to students' self-assessment } \\
\text { but not clear if graders were blinded to students' } \\
\text { identity }\end{array}$ \\
\hline Incomplete outcome data & Low & There are no missing data \\
\hline Free of selective reporting & Low & Data reported are consistent with study objectives \\
\hline
\end{tabular}

\begin{tabular}{|c|c|c|}
\hline \multicolumn{3}{|l|}{ Tuncer (NRS) } \\
\hline Entry & Risk & Description \\
\hline Selection of participants & Low & $\begin{array}{l}\text { All participants are from the same population and } \\
\text { data were collected prospectively. }\end{array}$ \\
\hline Confounding variables & Low & $\begin{array}{l}\text { Students initial ability is controlled by baseline } \\
\text { measurement. }\end{array}$ \\
\hline Measurement of exposure & Unclear & $\begin{array}{l}\text { Information limits to "course director calibrated } \\
\text { supervising faculty" }\end{array}$ \\
\hline $\begin{array}{l}\text { Blinding of outcome } \\
\text { assessment }\end{array}$ & Unclear & \\
\hline Incomplete outcome data & Low & There are no missing data \\
\hline Free of selective reporting & Low & Data reported are consistent with study objectives \\
\hline
\end{tabular}

\section{Radjaeipour (NRS)}

\begin{tabular}{|l|l|l|}
\hline Entry & Risk & Description \\
\hline Selection of participants & Low & All participants are from the same population and \\
\hline
\end{tabular}




\begin{tabular}{|l|l|l|}
\hline & & data were collected prospectively. \\
\hline Confounding variables & Unclear & \\
\hline Measurement of exposure & Low & Trained graders using standardise rubrics \\
\hline $\begin{array}{l}\text { Blinding of outcome } \\
\text { assessment }\end{array}$ & Low & Grading was performed anonymously \\
\hline Incomplete outcome data & Low & There is no missing data \\
\hline Free of selective reporting & Low & Data reported are consistent with study objectives \\
\hline
\end{tabular}

\begin{tabular}{|c|c|c|}
\hline \multicolumn{3}{|l|}{ de Peralta et al (NRS) } \\
\hline Entry & Risk & Description \\
\hline Selection of participants & Low & $\begin{array}{l}\text { All participants are from the same population and } \\
\text { data were collected prospectively. }\end{array}$ \\
\hline Confounding variables & Unclear & \\
\hline Measurement of exposure & Low & $\begin{array}{l}\text { Graders participated in calibration activities and } \\
\text { grading criteria is regularly updated. }\end{array}$ \\
\hline $\begin{array}{l}\text { Blinding of outcome } \\
\text { assessment }\end{array}$ & Unclear & \\
\hline Incomplete outcome data & Low & There is not missing data \\
\hline Free of selective reporting & Low & Data reported are consistent with study objectives \\
\hline
\end{tabular}

\begin{tabular}{|c|c|c|}
\hline \multicolumn{3}{|l|}{ Knight et al* (NRS) } \\
\hline Entry & Risk & Description \\
\hline Selection of participants & Low & $\begin{array}{l}\text { All participants are from the same population and } \\
\text { data were collected prospectively. }\end{array}$ \\
\hline Confounding variables & Low & $\begin{array}{l}\text { Students previous skills is disclosed. Investigators } \\
\text { did not find significant differences between the two } \\
\text { groups on entry GPA and academic records. }\end{array}$ \\
\hline Measurement of exposure & Low & Data measured by trained experts \\
\hline $\begin{array}{l}\text { Blinding of outcome } \\
\text { assessment }\end{array}$ & Unclear & \\
\hline Incomplete outcome data & Low & There is not missing data \\
\hline Free of selective reporting & Low & $\begin{array}{l}\text { Data reported is consistent with objectives and } \\
\text { methods. The same data as a previous study is } \\
\text { reported }\end{array}$ \\
\hline
\end{tabular}

This article is protected by copyright. All rights reserved 


\section{Geissler (NRS)}

\begin{tabular}{|l|l|l|}
\hline Entry & Risk & Description \\
\hline Selection of participants & Low & $\begin{array}{l}\text { All participants are from the same population and } \\
\text { data were collected prospectively. }\end{array}$ \\
\hline Confounding variables & High & $\begin{array}{l}\text { Confounding factors such students' general } \\
\text { capability are not reported }\end{array}$ \\
\hline Measurement of exposure & Low & Data measured by trained experts using rubrics \\
\hline $\begin{array}{l}\text { Blinding of outcome } \\
\text { assessment }\end{array}$ & Unclear & $\begin{array}{l}\text { There is not missing data } \\
\text { methods. The same data as a previous study is } \\
\text { Incomplete outcome data }\end{array}$ \\
\hline Free of selective reporting & Low & \\
\hline
\end{tabular}

1. Kim SY, Park JE, Lee YJ, et al. Testing a tool for assessing the risk of bias for nonrandomized studies showed moderate reliability and promising validity. J Clin Epidemiol. 2013;66(4):408414.

This article is protected by copyright. All rights reserved 
Appendix 3: Excluded articles and reasons of exclusion.

\begin{tabular}{|c|c|c|}
\hline Author (first) & Title & Reason for exclusion \\
\hline Roque et al ${ }^{1}$ & $\begin{array}{l}\text { Assessment of critical analysis } \\
\text { competency in first year dentistry } \\
\text { students using a self-evaluation tool }\end{array}$ & $\begin{array}{l}\text { Does not include } \\
\text { psychomotor skills }\end{array}$ \\
\hline Best and Messer ${ }^{2}$ & $\begin{array}{l}\text { Effectiveness of interventions to } \\
\text { promote continuing professional } \\
\text { development for dentists }\end{array}$ & $\begin{array}{l}\text { Does not include } \\
\text { psychomotor skills }\end{array}$ \\
\hline Matheos et $\mathrm{al}^{3}$ & $\begin{array}{l}\text { The effects of an interactive } \\
\text { software application on the self- } \\
\text { assessment ability of dental } \\
\text { students }\end{array}$ & $\begin{array}{l}\text { Does not include } \\
\text { psychomotor skills }\end{array}$ \\
\hline Moni et $\mathrm{al}^{4}$ & $\begin{array}{l}\text { Using student feedback to construct } \\
\text { an assessment rubric for a concept } \\
\text { map in physiology }\end{array}$ & $\begin{array}{l}\text { Does not include } \\
\text { psychomotor skills }\end{array}$ \\
\hline Zijlstra-Shaw $^{5}$ & $\begin{array}{l}\text { Assessment of professional } \\
\text { behaviour - A comparison of self- } \\
\text { assessment by first year dental } \\
\text { students and assessment by staff }\end{array}$ & $\begin{array}{l}\text { Does not include } \\
\text { psychomotor skills }\end{array}$ \\
\hline Broder \& Janal ${ }^{6}$ & $\begin{array}{l}\text { Promoting interpersonal skills and } \\
\text { cultural sensitivity among dental } \\
\text { students }\end{array}$ & $\begin{array}{l}\text { Does not include } \\
\text { psychomotor skills }\end{array}$ \\
\hline Keselyak et al $^{7}$ & $\begin{array}{l}\text { Evaluation of Group Assessment in } \\
\text { a Didactic Dental Hygiene Course }\end{array}$ & $\begin{array}{l}\text { Does not include } \\
\text { psychomotor skills }\end{array}$ \\
\hline Cox et $\mathrm{al}^{8}$ & $\begin{array}{l}\text { Making a mark--taking assessment } \\
\text { to technology }\end{array}$ & $\begin{array}{l}\text { Does not include } \\
\text { psychomotor skills }\end{array}$ \\
\hline González et al $^{9}$ & $\begin{array}{l}\text { A web-based learning tool } \\
\text { improves student performance in } \\
\text { statistics: A randomized masked } \\
\text { trial }\end{array}$ & $\begin{array}{l}\text { Does not include } \\
\text { psychomotor skills }\end{array}$ \\
\hline Hay et $\mathrm{al}^{10}$ & $\begin{array}{l}\text { Non-traditional learners in higher } \\
\text { education: comparison of a } \\
\text { traditional MCQ examination with } \\
\text { concept mapping to assess learning }\end{array}$ & $\begin{array}{l}\text { Does not include } \\
\text { psychomotor skills }\end{array}$ \\
\hline
\end{tabular}

This article is protected by copyright. All rights reserved 


\begin{tabular}{|c|c|c|}
\hline & $\begin{array}{l}\text { in a dental radiological science } \\
\text { course }\end{array}$ & \\
\hline Matthews ${ }^{11}$ & $\begin{array}{l}\text { Multifaceted strategy needed to } \\
\text { improve dentists' adherence to } \\
\text { evidence-based guidelines }\end{array}$ & $\begin{array}{l}\text { Does not include } \\
\text { psychomotor skills }\end{array}$ \\
\hline Grieveson et al ${ }^{12}$ & $\begin{array}{l}\text { Evaluation of workplace based } \\
\text { assessment tools in dental } \\
\text { foundation training }\end{array}$ & $\begin{array}{l}\text { Does not include } \\
\text { psychomotor skills }\end{array}$ \\
\hline Holmes et al ${ }^{13}$ & $\begin{array}{l}\text { Developing an assessment in dental } \\
\text { public health for clinical } \\
\text { undergraduates attending a primary } \\
\text { dental care outreach programme }\end{array}$ & $\begin{array}{l}\text { Does not include } \\
\text { psychomotor skills }\end{array}$ \\
\hline Jackson \& Murff ${ }^{14}$ & $\begin{array}{l}\text { Effectively teaching self- } \\
\text { assessment: preparing the dental } \\
\text { hygiene student to provide quality } \\
\text { care }\end{array}$ & $\begin{array}{l}\text { Does not include } \\
\text { psychomotor skills }\end{array}$ \\
\hline Barnes et al ${ }^{15}$ & $\begin{array}{l}\text { Development of the Maturity } \\
\text { Matrix Dentistry (MMD): A } \\
\text { primary care dental team } \\
\text { development tool }\end{array}$ & $\begin{array}{l}\text { Does not include } \\
\text { psychomotor skills }\end{array}$ \\
\hline Edo et $\mathrm{al}^{16}$ & $\begin{array}{l}\text { Web-based learning in } \\
\text { undergraduate dental education: } \\
\text { Bite-wing X-rays in paediatric } \\
\text { patients }\end{array}$ & $\begin{array}{l}\text { Does not include } \\
\text { psychomotor skills }\end{array}$ \\
\hline Priya et al ${ }^{17}$ & $\begin{array}{l}\text { Continuous Assessment of } \\
\text { Undergraduate Students at a Dental } \\
\text { College in India }\end{array}$ & $\begin{array}{l}\text { Does not include } \\
\text { psychomotor skills }\end{array}$ \\
\hline Sopka et $\mathrm{al}^{18}$ & $\begin{array}{l}\text { Practical skills training influences } \\
\text { knowledge and attitude of dental } \\
\text { students towards emergency } \\
\text { medical care }\end{array}$ & $\begin{array}{l}\text { Does not include } \\
\text { psychomotor skills }\end{array}$ \\
\hline Zijlstra-Shaw et al ${ }^{19}$ & $\begin{array}{l}\text { Assessing professionalism within } \\
\text { dental education; the need for a } \\
\text { definition }\end{array}$ & $\begin{array}{l}\text { Does not include } \\
\text { psychomotor skills }\end{array}$ \\
\hline Haj-Ali \& Al Quran ${ }^{20}$ & $\begin{array}{l}\text { Team-based learning in a } \\
\text { preclinical removable denture }\end{array}$ & $\begin{array}{l}\text { Does not include } \\
\text { psychomotor skills }\end{array}$ \\
\hline
\end{tabular}

This article is protected by copyright. All rights reserved 


\begin{tabular}{|c|c|c|}
\hline & \multicolumn{2}{|l|}{ prosthesis module in a United Arab } \\
\hline & Emirates dental school & \\
\hline Jonas-Dwyer \& Boyd ${ }^{21}$ & $\begin{array}{l}\text { First reflections: third-year } \\
\text { dentistry students' introduction to } \\
\text { reflective practice }\end{array}$ & $\begin{array}{l}\text { Does not include } \\
\text { psychomotor skills }\end{array}$ \\
\hline Winning et $\mathrm{al}^{22}$ & $\begin{array}{l}\text { Validity of scores from } \\
\text { communication skills instruments } \\
\text { for patients and their dental student- } \\
\text { clinicians }\end{array}$ & $\begin{array}{l}\text { Does not include } \\
\text { psychomotor skills }\end{array}$ \\
\hline Gatumu et $\mathrm{al}^{23}$ & $\begin{array}{l}\text { Evaluation of usage of virtual } \\
\text { microscopy for the study of } \\
\text { histology in the medical, dental, } \\
\text { and veterinary undergraduate } \\
\text { programs of a UK University }\end{array}$ & $\begin{array}{l}\text { Does not include } \\
\text { psychomotor skills }\end{array}$ \\
\hline Leisnert $^{24}$ & $\begin{array}{l}\text { Self-directed learning. teamwork, } \\
\text { holistic view and oral health }\end{array}$ & $\begin{array}{l}\text { Does not include } \\
\text { psychomotor skills }\end{array}$ \\
\hline Prior et $\mathrm{al}^{25}$ & $\begin{array}{l}\text { Evaluating an audit and feedback } \\
\text { intervention for reducing antibiotic } \\
\text { prescribing behaviour in general } \\
\text { dental practice (the RAPiD trial): A } \\
\text { partial factorial cluster randomised } \\
\text { trial protocol }\end{array}$ & $\begin{array}{l}\text { Does not include } \\
\text { psychomotor skills }\end{array}$ \\
\hline Sampaio-Maia et $\mathrm{al}^{26}$ & $\begin{array}{l}\text { Wiki as a tool for Microbiology } \\
\text { teaching, learning and assessment }\end{array}$ & $\begin{array}{l}\text { Does not include } \\
\text { psychomotor skills }\end{array}$ \\
\hline Yoshida et $\mathrm{al}^{27}$ & $\begin{array}{l}\text { Effectiveness of a simulated patient } \\
\text { training programme based on } \\
\text { trainee response accuracy and } \\
\text { appropriateness of feedback }\end{array}$ & $\begin{array}{l}\text { Does not include } \\
\text { psychomotor skills }\end{array}$ \\
\hline Al-Ansari \& El Tautawi ${ }^{28}$ & $\begin{array}{l}\text { Comparison of Three Evidence- } \\
\text { Based Practice Learning } \\
\text { Assessment Methods in Dental } \\
\text { Curricula }\end{array}$ & $\begin{array}{l}\text { Does not include } \\
\text { psychomotor skills }\end{array}$ \\
\hline Al-Khotani et $\mathrm{al}^{29}$ & $\begin{array}{l}\text { Study on self-assessment regarding } \\
\text { knowledge of temporomandibular } \\
\text { disorders in children/adolescents by } \\
\text { Swedish and Saudi Arabian dentists }\end{array}$ & $\begin{array}{l}\text { Does not include } \\
\text { psychomotor skills }\end{array}$ \\
\hline
\end{tabular}

This article is protected by copyright. All rights reserved 


\begin{tabular}{|c|c|c|}
\hline Al-Rawi et al ${ }^{30}$ & $\begin{array}{l}\text { Development of a mobile device } \\
\text { optimized cross platform- } \\
\text { compatible oral pathology and } \\
\text { radiology spaced repetition system } \\
\text { for dental education }\end{array}$ & $\begin{array}{l}\text { Does not include } \\
\text { psychomotor skills }\end{array}$ \\
\hline Alfaris et $\mathrm{al}^{31}$ & $\begin{array}{l}\text { A one-day dental faculty workshop } \\
\text { in writing multiple-choice } \\
\text { questions: An impact evaluation }\end{array}$ & $\begin{array}{l}\text { Does not include } \\
\text { psychomotor skills }\end{array}$ \\
\hline El Tantawi et $\mathrm{al}^{32}$ & $\begin{array}{l}\text { E-Assessment in a limited- } \\
\text { resources dental school using an } \\
\text { open-source learning management } \\
\text { system }\end{array}$ & $\begin{array}{l}\text { Does not include } \\
\text { psychomotor skills }\end{array}$ \\
\hline $\begin{array}{l}\text { Sanchez_Sanhueza \& } \\
\text { Cabrera }^{33}\end{array}$ & $\begin{array}{l}\text { Praxis teaching in the ambit of } \\
\text { learning assessment of endodontics } \\
\text { in a Chilean university }\end{array}$ & $\begin{array}{l}\text { Does not include } \\
\text { psychomotor skills }\end{array}$ \\
\hline Freda \& Lipp $^{34}$ & $\begin{array}{l}\text { Test-Enhanced Learning in } \\
\text { Competence Based Predoctoral } \\
\text { Orthodontics: A Four-Year Study }\end{array}$ & $\begin{array}{l}\text { Does not include } \\
\text { psychomotor skills }\end{array}$ \\
\hline van Schaik et $\mathrm{al}^{35}$ & $\begin{array}{l}\text { Perceptions of Peer-to-Peer } \\
\text { Interprofessional Feedback Among } \\
\text { Students in the Health Professions }\end{array}$ & $\begin{array}{l}\text { Does not include } \\
\text { psychomotor skills }\end{array}$ \\
\hline Wong et $\mathrm{al}^{36}$ & $\begin{array}{l}\text { Measuring interprofessional } \\
\text { competencies and attitudes among } \\
\text { health professional students } \\
\text { creating family planning virtual } \\
\text { patient cases }\end{array}$ & $\begin{array}{l}\text { Does not include } \\
\text { psychomotor skills }\end{array}$ \\
\hline Arias et $\mathrm{al}^{37}$ & $\begin{array}{l}\text { New curricular design in } \\
\text { biostatistics to prepare residents for } \\
\text { an evidence-based practice and } \\
\text { lifelong learning education: a pilot } \\
\text { approach }\end{array}$ & $\begin{array}{l}\text { Does not include } \\
\text { psychomotor skills }\end{array}$ \\
\hline Hong \& Plugge ${ }^{38}$ & $\begin{array}{l}\text { Critical appraisal skills teaching in } \\
\text { UK dental schools }\end{array}$ & $\begin{array}{l}\text { Does not include } \\
\text { psychomotor skills }\end{array}$ \\
\hline Lipp et $\mathrm{al}^{39}$ & $\begin{array}{l}\text { Types of Feedback in Competency- } \\
\text { Based Predoctoral Orthodontics: } \\
\text { Effects on Students' Attitudes and }\end{array}$ & $\begin{array}{l}\text { Does not include } \\
\text { psychomotor skills }\end{array}$ \\
\hline
\end{tabular}

This article is protected by copyright. All rights reserved 


\begin{tabular}{|c|c|c|}
\hline & Confidence & \\
\hline Partido $^{40}$ & $\begin{array}{l}\text { Dental Hygiene Students' Self- } \\
\text { Assessment of Ergonomics } \\
\text { Utilizing Photography }\end{array}$ & $\begin{array}{l}\text { Does not include } \\
\text { psychomotor skills }\end{array}$ \\
\hline Zijlstra-Shaw et al ${ }^{41}$ & $\begin{array}{l}\text { Evaluation of an assessment system } \\
\text { for professionalism amongst dental } \\
\text { students }\end{array}$ & $\begin{array}{l}\text { Does not include } \\
\text { psychomotor skills }\end{array}$ \\
\hline Morales et $\mathrm{al}^{42}$ & $\begin{array}{l}\text { Validation of a self-assessment tool } \\
\text { to measure critical analysis } \\
\text { competency in first year dentistry } \\
\text { students }\end{array}$ & $\begin{array}{l}\text { Does not include } \\
\text { psychomotor skills, only } \\
\text { abstract available }\end{array}$ \\
\hline Gonzalez-Cabezas et $\mathrm{al}^{43}$ & $\begin{array}{l}\text { Association between dental student- } \\
\text { developed exam questions and } \\
\text { learning at higher cognitive levels }\end{array}$ & $\begin{array}{l}\text { Does not include } \\
\text { psychomotor skills. }\end{array}$ \\
\hline Öcek et al ${ }^{44}$ & $\begin{array}{l}\text { Development of a rational } \\
\text { antibiotic usage course for dentists }\end{array}$ & $\begin{array}{l}\text { Does not include } \\
\text { psychomotor skills. Course } \\
\text { development }\end{array}$ \\
\hline Asadoorian \& Batty ${ }^{45}$ & $\begin{array}{l}\text { An evidence-based model of } \\
\text { effective self-assessment for } \\
\text { directing professional learning }\end{array}$ & $\begin{array}{l}\text { Does not include } \\
\text { psychomotor skills, } \\
\text { description of methodology }\end{array}$ \\
\hline Clark et $\mathrm{al}^{46}$ & $\begin{array}{l}\text { 'On Track' - An educational } \\
\text { resource to support dental SHO } \\
\text { training }\end{array}$ & $\begin{array}{l}\text { Evaluation of an educational } \\
\text { resource for dental Senior } \\
\text { Health Officer. Broad } \\
\text { educational resource beyond } \\
\text { psychomotor skills }\end{array}$ \\
\hline Davenport et $\mathrm{al}^{47}$ & $\begin{array}{l}\text { Learning throughout life: can a } \\
\text { progress file help? }\end{array}$ & $\begin{array}{l}\text { Feedback on a lifelong } \\
\text { learning tool, does not } \\
\text { include psychomotor skills }\end{array}$ \\
\hline Kournetas et al ${ }^{48}$ & $\begin{array}{l}\text { Assessing the reliability of a digital } \\
\text { preparation assistant system used in } \\
\text { dental education }\end{array}$ & Does not include students \\
\hline Haj-Ali \& Feil ${ }^{49}$ & $\begin{array}{l}\text { Rater reliability: short-and long- } \\
\text { term effects of calibration training }\end{array}$ & Does not include students \\
\hline Sharaf et $\mathrm{al}^{50}$ & $\begin{array}{l}\text { Intra-and inter-examiner variability } \\
\text { in evaluating preclinical pediatric } \\
\text { dentistry operative procedures }\end{array}$ & Does not include students \\
\hline
\end{tabular}

This article is protected by copyright. All rights reserved 


\begin{tabular}{|c|c|c|}
\hline McAndrew \& Pierre ${ }^{51}$ & $\begin{array}{l}\text { Using multiple measures to } \\
\text { evaluate a dental faculty } \\
\text { development program }\end{array}$ & Does not include students \\
\hline Renne et $\mathrm{al}^{52}$ & $\begin{array}{l}\text { E4D compare software: an } \\
\text { alternative to faculty grading in } \\
\text { dental education }\end{array}$ & Does not include students \\
\hline AlHumaid et $\mathrm{al}^{53}$ & $\begin{array}{l}\text { Agreement in Scoring Preclinical } \\
\text { Dental Procedures: Impact on } \\
\text { Grades and Instructor-Related } \\
\text { Determinants }\end{array}$ & Does not include students \\
\hline Garland \& Newell ${ }^{54}$ & $\begin{array}{l}\text { Dental hygiene faculty calibration } \\
\text { in the evaluation of calculus } \\
\text { detection }\end{array}$ & $\begin{array}{l}\text { Does not involve dental } \\
\text { students }\end{array}$ \\
\hline Schmitt et al ${ }^{55}$ & $\begin{array}{l}\text { Study on the Interrater Reliability } \\
\text { of an OSPE (Objective Structured } \\
\text { Practical Examination) - Subject to } \\
\text { the Evaluation Mode in the } \\
\text { Phantom Course of Operative } \\
\text { Dentistry }\end{array}$ & $\begin{array}{l}\text { Does not involve dental } \\
\text { students }\end{array}$ \\
\hline Jenkins et al ${ }^{56}$ & $\begin{array}{l}\text { Evaluating undergraduate } \\
\text { preclinical operative skill; use of a } \\
\text { glance and grade marking system }\end{array}$ & Does not include students \\
\hline Fitzgerald et $\mathrm{al}^{57}$ & $\begin{array}{l}\text { A longitudinal study of self- } \\
\text { assessment accuracy }\end{array}$ & $\begin{array}{l}\text { Does not include dental } \\
\text { students }\end{array}$ \\
\hline Gordon $\mathrm{M}^{58}$ & $\begin{array}{l}\text { A review of the validity and } \\
\text { accuracy of self-assessments in } \\
\text { health professions training }\end{array}$ & $\begin{array}{l}\text { Does not include dental } \\
\text { students }\end{array}$ \\
\hline Thammasitboon et al ${ }^{59}$ & $\begin{array}{l}\text { Problem-based learning at the } \\
\text { Harvard school of dental medicine: } \\
\text { Self-assessment of performance in } \\
\text { postdoctoral training }\end{array}$ & Mostly clinical \\
\hline $\begin{array}{l}\text { Pileggi, Roberta Glickman, } \\
\text { Gerald N. }\end{array}$ & $\begin{array}{l}\text { A cost-effective simulation } \\
\text { curriculum for preclinical } \\
\text { endodontics }\end{array}$ & $\begin{array}{l}\text { Description of new teaching } \\
\text { method }\end{array}$ \\
\hline McAndrew et al ${ }^{61}$ & $\begin{array}{l}\text { Characteristics of effective } \\
\text { simulation (preclinical) teachers as }\end{array}$ & $\begin{array}{l}\text { Not an assessment } \\
\text { intervention }\end{array}$ \\
\hline
\end{tabular}

This article is protected by copyright. All rights reserved 


\begin{tabular}{|c|c|c|}
\hline & $\begin{array}{l}\text { identified by dental students: A } \\
\text { qualitative study }\end{array}$ & \\
\hline Mays \& Branch-Mays ${ }^{62}$ & $\begin{array}{l}\text { A Systematic Review of the Use of } \\
\text { Self-Assessment in Preclinical and } \\
\text { Clinical Dental Education }\end{array}$ & $\begin{array}{l}\text { Not an intervention but } \\
\text { literature review }\end{array}$ \\
\hline de Azevedo et al ${ }^{63}$ & $\begin{array}{l}\text { Comparative Effectiveness of } \\
\text { Dental Anatomy Carving } \\
\text { Pedagogy: A Systematic Review }\end{array}$ & Not an intervention. Lit rev \\
\hline Postma \& White ${ }^{64}$ & $\begin{array}{l}\text { Developing clinical reasoning in } \\
\text { the classroom - analysis of the } 4 \mathrm{C} / \\
\text { ID-model }\end{array}$ & Not an intervention \\
\hline Matheos et $\mathrm{al}^{65}$ & $\begin{array}{l}\text { Assessment of knowledge and } \\
\text { competencies related to implant } \\
\text { dentistry in undergraduate and } \\
\text { postgraduate university education }\end{array}$ & $\begin{array}{l}\text { This is not an intervention } \\
\text { but investigates and } \\
\text { describes assessments } \\
\text { methodologies for } \\
\text { implantology }\end{array}$ \\
\hline Firmstone et al ${ }^{66}$ & $\begin{array}{l}\text { Systematic review of the } \\
\text { effectiveness of continuing dental } \\
\text { professional development on } \\
\text { learning, behavior, or patient } \\
\text { outcomes }\end{array}$ & $\begin{array}{l}\text { This is not an intervention } \\
\text { but literature review on CPD } \\
\text { effectiveness }\end{array}$ \\
\hline Hauser \& Bowen ${ }^{67}$ & $\begin{array}{l}\text { Primer on Preclinical Instruction } \\
\text { and Evaluation.pdf }\end{array}$ & $\begin{array}{l}\text { This not an intervention but } \\
\text { a review of the literature }\end{array}$ \\
\hline $\begin{array}{l}\text { Nance, Elizabeth T Lanning, } \\
\text { Sharon K Gunsolley, John } \\
\text { C }^{68}\end{array}$ & $\begin{array}{l}\text { Dental anatomy carving computer- } \\
\text { assisted instruction program: an } \\
\text { assessment of student performance } \\
\text { and perceptions }\end{array}$ & $\begin{array}{l}\text { Compares traditional } \\
\text { delivery vs computer } \\
\text { assisted }\end{array}$ \\
\hline $\begin{array}{l}\text { Dantas, Alessandra K. } \\
\text { Shinagawa, Adriana Deboni, } \\
\text { Maria Cristina Z. }\end{array}$ & $\begin{array}{l}\text { Assessment of Preclinical Learning } \\
\text { on Oral Surgery Using Three } \\
\text { Instructional Strategies }\end{array}$ & $\begin{array}{l}\text { Evaluation of three teaching } \\
\text { methods rather than } \\
\text { assessment feedback } \\
\text { intervention }\end{array}$ \\
\hline 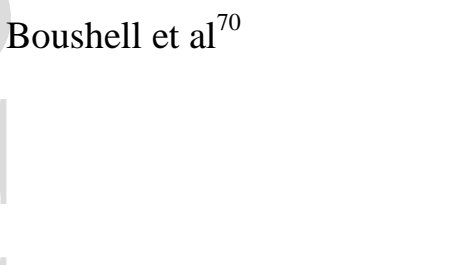 & $\begin{array}{l}\text { Learn-A-Prep II as a predictor of } \\
\text { psychomotor performance in a } \\
\text { restorative dentistry course }\end{array}$ & $\begin{array}{l}\text { Investigate the potential of } \\
\text { Learn-A prep to early } \\
\text { diagnose students' } \\
\text { psychomotor skills }\end{array}$ \\
\hline Polyzois et $\mathrm{al}^{71}$ & Can evaluation of a dental & Investigate the effectiveness \\
\hline
\end{tabular}

This article is protected by copyright. All rights reserved 


\begin{tabular}{|c|c|c|}
\hline & $\begin{array}{l}\text { procedure at the outset of learning } \\
\text { predict later performance at the } \\
\text { preclinical level? A pilot study }\end{array}$ & $\begin{array}{l}\text { of conventional pre-clinical } \\
\text { training }\end{array}$ \\
\hline $\begin{array}{l}\text { Gadbury-Amyot, Cynthia C } \\
\text { Purk, John H Williams, } \\
\text { Brian Joseph Van Ness, } \\
\text { Christopher J. }^{72}\end{array}$ & $\begin{array}{l}\text { Using tablet technology and } \\
\text { instructional videos to enhance } \\
\text { preclinical dental laboratory } \\
\text { learning }\end{array}$ & $\begin{array}{l}\text { Investigate the impact of } \\
\text { tablet technology with } \\
\text { accompanying instructional } \\
\text { videos on teaching and } \\
\text { learning outcomes in a } \\
\text { preclinical dental laboratory } \\
\text { setting }\end{array}$ \\
\hline Bedi et $\mathrm{al}^{73}$ & $\begin{array}{l}\text { Agreement of gradin using pictorial } \\
\text { grading criteria }\end{array}$ & $\begin{array}{l}\text { Did not involve } \\
\text { psychomotor skills }\end{array}$ \\
\hline $\begin{array}{l}\text { Morrow, Jay A Pulido, M } \\
\text { Teresa Smith, P Bradford } \\
\text { McDaniel, Thomas F } \\
\text { Willcox, Austin B }{ }^{74}\end{array}$ & $\begin{array}{l}\text { Effective use of e-grading in the } \\
\text { dental simulation clinic }\end{array}$ & $\begin{array}{l}\text { Describe the development } \\
\text { and implementation of a } \\
\text { grading software }\end{array}$ \\
\hline Barrero et al ${ }^{75}$ & $\begin{array}{l}\text { Dental Students' Perceived } \\
\text { Preparedness to Treat Patients in } \\
\text { Clinic After a Fixed Prosthodontics } \\
\text { Course: Survey Results of a Case } \\
\text { Study }\end{array}$ & $\begin{array}{l}\text { Not assessment feedback } \\
\text { involved }\end{array}$ \\
\hline $\begin{array}{l}\text { Schuster, G. M. Hunt, R. J. } \\
\text { Haering, H. J. }^{76}\end{array}$ & $\begin{array}{l}\text { Effect of a Pilot Preclinical } \\
\text { Incentive Program on Dental } \\
\text { Students' Performance on a } \\
\text { Clinical Competency Exam }\end{array}$ & $\begin{array}{l}\text { Intervention is not on } \\
\text { assessment feedback }\end{array}$ \\
\hline $\begin{array}{l}\text { Shugars, D. A. May, K. N. } \\
\text { Vann, W. F. }^{77}\end{array}$ & $\begin{array}{l}\text { Comprehensive evaluation in a } \\
\text { preclinical restorative dentistry } \\
\text { technique course }\end{array}$ & $\begin{array}{l}\text { Description of evaluation } \\
\text { system }\end{array}$ \\
\hline $\begin{array}{l}\text { Wiegman, JE van de Poel, } \\
\text { FCM Purdell-Lewis, } \mathrm{D}^{78}\end{array}$ & $\begin{array}{l}\text { The value of an assessment system } \\
\text { in a preclinical amalgam exercise }\end{array}$ & $\begin{array}{l}\text { Description of evaluation } \\
\text { system (to see amalgam } \\
\text { adaptation) }\end{array}$ \\
\hline Abrams, RG Kelley, ML ${ }^{79}$ & $\begin{array}{l}\text { Student self-evaluation in a } \\
\text { pediatric-operative technique } \\
\text { course }\end{array}$ & $\begin{array}{l}\text { Description of evaluation } \\
\text { system }\end{array}$ \\
\hline $\begin{array}{l}\text { Edwards, W. S. Morse, P. K. } \\
\text { Mitchell, R. J. }\end{array}$ & $\begin{array}{l}\text { A practical evaluation system for } \\
\text { preclinical restorative dentistry }\end{array}$ & $\begin{array}{l}\text { Description of evaluation } \\
\text { system }\end{array}$ \\
\hline
\end{tabular}

This article is protected by copyright. All rights reserved 


\begin{tabular}{|c|c|c|}
\hline $\begin{array}{l}\text { Forehand, Lena S Vann, WF } \\
\text { Shugars, } \text { DA }^{81}\end{array}$ & $\begin{array}{l}\text { Student self-evaluation in } \\
\text { preclinical restorative dentistry }\end{array}$ & $\begin{array}{l}\text { Description of evaluation } \\
\text { system does not compare } \\
\text { groups }\end{array}$ \\
\hline $\begin{array}{l}\text { Cho, George C Chee, } \\
\text { Winston WL Tan, Derek T }{ }^{82}\end{array}$ & $\begin{array}{l}\text { Dental Students' Ability to } \\
\text { Evaluate Themselves in Fixed } \\
\text { Prosthodontics" }\end{array}$ & Description \\
\hline $\begin{array}{l}\text { San Diego, JP Newton, T } \\
\text { Quinn, BFA Cox, MJ } \\
\text { Woolford, } \text { MJ }^{83}\end{array}$ & $\begin{array}{l}\text { Levels of agreement between } \\
\text { student and staff assessments of } \\
\text { clinical skills in performing cavity } \\
\text { preparation in artificial teeth }\end{array}$ & Description \\
\hline Denehy, GE Fuller, JL ${ }^{84}$ & $\begin{array}{l}\text { Student peer evaluation: an adjunct } \\
\text { to preclinical laboratory evaluation }\end{array}$ & Description \\
\hline Brehm, TW $^{85}$ & $\begin{array}{l}\text { Peer evaluation of fixed partial } \\
\text { dentures in a preclinical course }\end{array}$ & Description \\
\hline $\begin{array}{l}\text { Satterthwaite, J. D. Grey, N. } \\
\text { J. A. }{ }^{86}\end{array}$ & $\begin{array}{l}\text { Peer-group assessment of pre- } \\
\text { clinical operative skills in } \\
\text { restorative dentistry and } \\
\text { comparison with experienced } \\
\text { assessors }\end{array}$ & Description \\
\hline $\begin{array}{l}\text { Olson, PC Comet-Epstein, S } \\
87\end{array}$ & $\begin{array}{l}\text { Increasing objectivity in evaluation } \\
\text { for a preclinical technique course }\end{array}$ & Description \\
\hline $\begin{array}{l}\text { Karl, M. Graef, F. } \\
\text { Wichmann, M. Beck, N. }{ }^{88}\end{array}$ & $\begin{array}{l}\text { Evaluation of tooth preparations - A } \\
\text { comparative study between faculty } \\
\text { members and pre-clinical students }\end{array}$ & Description \\
\hline Cohen, S. Silverstri, A ${ }^{89}$ & $\begin{array}{l}\text { Evaluation of describing and } \\
\text { grading preclinical technical } \\
\text { exercises }\end{array}$ & Description \\
\hline Bazan, MT Seale, NS ${ }^{90}$ & $\begin{array}{l}\text { A technique for immediate } \\
\text { evaluation of preclinical exercises }\end{array}$ & Description \\
\hline Nick, Doyle et al ${ }^{91}$ & $\begin{array}{l}\text { The ability of dental students and } \\
\text { faculty to estimate the total occlusal } \\
\text { convergence of prepared teeth }\end{array}$ & Description \\
\hline $\begin{array}{l}\text { Foley, J. I. Richardson, G. L. } \\
\text { Drummie, J. }{ }^{92}\end{array}$ & $\begin{array}{l}\text { Agreement Among Dental } \\
\text { Students, Peer Assessors, and Tutor } \\
\text { in Assessing Students' Competence } \\
\text { in Preclinical Skills }\end{array}$ & Description \\
\hline
\end{tabular}




\begin{tabular}{|c|c|c|}
\hline ee, C.et $\mathrm{al}^{93}$ & $\begin{array}{l}\text { The Relationship Between Dental } \\
\text { Students' Assessment Ability and } \\
\text { Preclinical and Academic } \\
\text { Performance in Operative Dentistry }\end{array}$ & Description \\
\hline Puryer \& O'Sullivan ${ }^{94}$ & $\begin{array}{l}\text { An introduction to standard setting } \\
\text { methods in dentistry }\end{array}$ & $\begin{array}{l}\text { Curriculum development not } \\
\text { an intervention }\end{array}$ \\
\hline Marshall et al ${ }^{95}$ & $\begin{array}{l}\text { Evidence-based dentistry: } \\
\text { assessment to document } \\
\text { progression to proficiency }\end{array}$ & $\begin{array}{l}\text { Curriculum development not } \\
\text { an intervention }\end{array}$ \\
\hline Field et al ${ }^{96}$ & $\begin{array}{l}\text { The Graduating European Dentist: } \\
\text { A New Undergraduate Curriculum } \\
\text { Framework INTRODUCTION }\end{array}$ & $\begin{array}{l}\text { Not an assessment } \\
\text { intervention }\end{array}$ \\
\hline Gerhard-Szep et al ${ }^{97}$ & $\begin{array}{l}\text { Assessment formats in dental } \\
\text { medicine: An overview }\end{array}$ & $\begin{array}{l}\text { Not an intervention but } \\
\text { review of assessments in } \\
\text { dentistry }\end{array}$ \\
\hline Feil ${ }^{98}$ & $\begin{array}{l}\text { Designing preclinical instruction } \\
\text { for psychomotor skills (I)-- } \\
\text { theoretical foundations of motor } \\
\text { skill performance and their } \\
\text { applications to dental education }\end{array}$ & There is not an intervention \\
\hline Chambers and Gissberger ${ }^{99}$ & $\begin{array}{l}\text { Toward a competency analysis of } \\
\text { operative dentistry technique skills }\end{array}$ & There is not an intervention \\
\hline Manogue et al ${ }^{100}$ & $\begin{array}{l}2.1 \text { Evolving methods of } \\
\text { assessment }\end{array}$ & There is not an intervention \\
\hline Knight ${ }^{101}$ & Toward faculty calibration & $\begin{array}{l}\text { There are not students } \\
\text { involved }\end{array}$ \\
\hline Kavadella et al ${ }^{102}$ & $\begin{array}{l}\text { Recommendations for the } \\
\text { development of e-modules for the } \\
\text { continuing professional } \\
\text { development of European dentists } \\
\text { Recommendations for the } \\
\text { development of e-modules for the } \\
\text { continuing professional } \\
\text { development of European dentists }\end{array}$ & This is not an intervention \\
\hline Kramer et $\mathrm{al}^{103}$ & Dental student assessment toolbox & $\begin{array}{l}\text { This is not an intervention } \\
\text { but a description of multiple }\end{array}$ \\
\hline
\end{tabular}

This article is protected by copyright. All rights reserved 
Moore \& Durham ${ }^{104}$

Redwood et al ${ }^{105}$

Council of European

Dentist $^{106}$

Licari et al ${ }^{107}$

Albino et al ${ }^{108}$

Feil \& Gatti ${ }^{109}$

Rhienmora, Phattanapon et $\mathrm{al}^{110}$

Kateeb, E. T. et al ${ }^{111}$

Quinn, Frank et $\mathrm{al}^{112}$

Callan, Richard S. et al ${ }^{113}$
Invited commentary: issues with assessing competence in

undergraduate dental education

The missing link: self-assessment

and continuing professional

development

Competences required for the

practice of dentistry in the

European Union

Designing evaluation forms to facilitate student learning

Assessing dental students'

competence: best practice

recommendations in the

performance assessment literature

and investigation of current

practices in predoctoral dental

education

Validation of a motor skills

performance theory with

applications for dental education

Intelligent dental training simulator

with objective skill assessment and

feedback

Utilising an innovative digital

software to grade pre-clinical

crown preparation exercise

A study comparing the

effectiveness of conventional

training and virtual reality

simulation in the skills acquisition

of junior dental students

Effect of employing different

typodonts when using E4D

Compare for dental student assessment methods

This is not an intervention

but a discussion on

assessments in dentistry

This is not an intervention

but a document of SA in

CPD context

This is not an intervention

but a document on

competencies

This is not an intervention but methodology description

This not an intervention on assessment feedback but an investigation on past and

current assessment

methodologies.

Validation of theory rather than intervention

Technology feedback

Technology feedback

Technology feedback

Technology feedback 
assessment

Taylor, C. L. Grey, N. J. A. A comparison of grades awarded by Technology feedback

Satterthwaite, J. D. ${ }^{114}$

peer assessment, faculty and a

digital scanning device in a pre-

clinical operative skills course

Callan, Richard S. et al ${ }^{115}$

Inter- and intra-rater reliability

Technology feedback

using different software versions of

E4D compare in dental education

Callan, Richard S. et al ${ }^{116}$

The validity of using E4D

Technology feedback

compare's "\% comparison" to

assess crown preparations in

preclinical dental education

Kikuchi, Hirono Ikeda,

Masaomi Araki, Koji ${ }^{117}$

Evaluation of a virtual reality

simulation system for porcelain

fused to metal crown preparation at

Tokyo medical and dental

University

Kwon, S. R. et al ${ }^{118}$

Dental anatomy grading:

Technology feedback

comparison between conventional

visual and a novel digital

assessment

Mays, K. A. Levine, E. ${ }^{119}$

Dental Students' Self-Assessment

Technology feedback

of Operative Preparations Using

CAD/CAM: A Preliminary

Analysis

Tiu, Janine et al ${ }^{120}$

Effectiveness of Crown Preparation

Technology feedback

Assessment Software As an

Educational Tool in Simulation

Clinic: A Pilot Study

Zou, Huiru et al ${ }^{121}$

A cavity preparation evaluation

Technology feedback

system in the skill assessment of

dental students

Esser, C. et al ${ }^{122}$

A comparison of the visual and

Technology feedback

technical assessment of

preparations made by dental

students

This article is protected by copyright. All rights reserved 


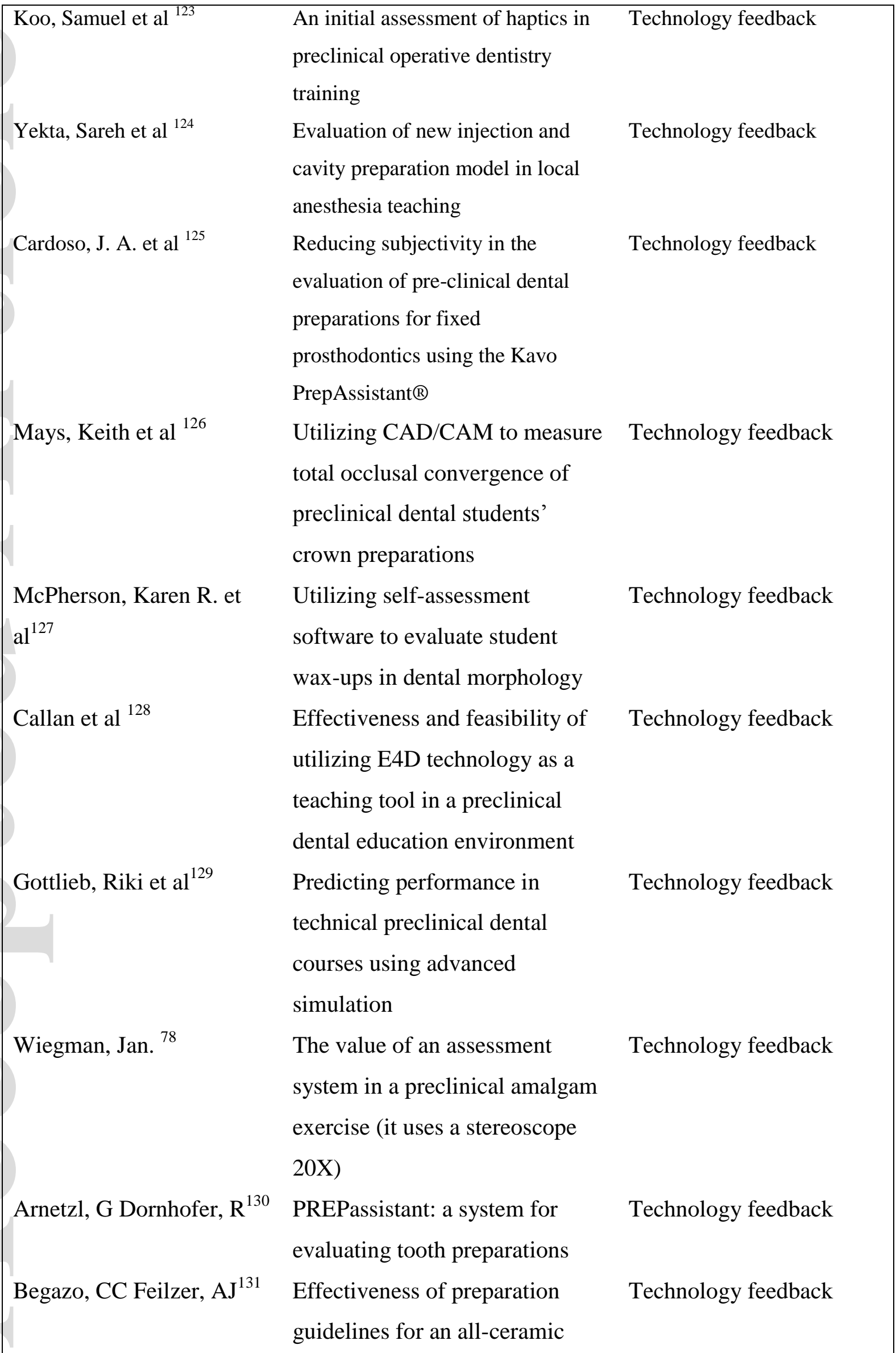

This article is protected by copyright. All rights reserved 
restorative system

Jasinevicius, T et al ${ }^{132}$

An evaluation of two dental

Technology feedback

simulation systems: virtual

reality versus contemporary non-

computer-assisted

Wierinck, Els et al ${ }^{133}$

Effect of augmented visual

Technology feedback

feedback from a virtual reality

simulation system on manual

dexterity training

Wierinck, Els et al ${ }^{134}$

Effect of reducing frequency of

Technology feedback

augmented feedback on manual

dexterity training and its

retention

Wierinck, Els et al ${ }^{135}$

Effect of tutorial input in

Technology feedback

addition to augmented feedback

on manual dexterity training and

its retention

Sharaf et al ${ }^{50}$

Intra-and inter-examiner

Technology feedback

variability in evaluating

preclinical paediatric dentistry

operative procedures

Sadid-Zadeh et al ${ }^{136}$

Effectiveness of virtual

Technology feedback

assessment

Kunkel et al ${ }^{137}$

The present study compared the

Technology feedback

subjective grading of student

tooth preparations by

experienced, well-calibrated

faculty with objective digital

grading of the same preparations

by means of the PrepCheck

software.

Mettes et $\mathrm{al}^{138}$

Routine oral examination:

Clinical setting 


\begin{tabular}{|c|c|c|}
\hline & $\begin{array}{l}\text { Clinical vignettes, a promising } \\
\text { tool for continuing professional } \\
\text { development? }\end{array}$ & \\
\hline $\mathrm{O}^{\prime}$ Sullivan et al ${ }^{139}$ & $\begin{array}{l}\text { The introduction of a new } \\
\text { assessment system in restorative } \\
\text { dentistry: The undergraduate and } \\
\text { patient experience }\end{array}$ & Clinical setting \\
\hline Ali, K et al ${ }^{140}$ & $\begin{array}{l}\text { Use of peer assessment in tooth } \\
\text { extraction competency }\end{array}$ & Clinical setting \\
\hline Bousdras, $\mathrm{V}$ et al ${ }^{141}$ & $\begin{array}{l}\text { Assessment of surgical skills in } \\
\text { implant dentistry }\end{array}$ & Clinical setting \\
\hline Woolley, S. et al ${ }^{142}$ & $\begin{array}{l}\text { A pilot study of the use and } \\
\text { perceived utility of a scale to } \\
\text { assess clinical dental teaching } \\
\text { within a UK dental school } \\
\text { restorative department }\end{array}$ & Clinical setting \\
\hline Chandrasekaran, S. et al ${ }^{143}$ & $\begin{array}{l}\text { Dental Students' Reflections on } \\
\text { Quality of Periodontal Care in } \\
\text { Dental School Clinics }\end{array}$ & Clinical setting \\
\hline $\begin{array}{l}\text { Karaharju-Suvanto, T. et } \\
\mathrm{al}^{144}\end{array}$ & $\begin{array}{l}\text { Gender differences in self- } \\
\text { assessed clinical competence-a } \\
\text { survey of young dentists in } \\
\text { Finland }\end{array}$ & Clinical setting \\
\hline Redford, R. et al ${ }^{145}$ & $\begin{array}{l}\text { Student perceptions of exodontia } \\
\text { competency assessment in a UK } \\
\text { dental school }\end{array}$ & Clinical setting \\
\hline
\end{tabular}

This article is protected by copyright. All rights reserved 
Sanderson, $\mathrm{T}^{146}$

Redwood, C. et al ${ }^{147}$

Gonzalez, B et al ${ }^{148}$

Kirkup, M. et al ${ }^{149}$

Gordon, $\mathrm{N}^{150}$

Lin, $\mathrm{C}$ et al ${ }^{151}$

Prescott-Clements, $\mathrm{L}^{152}$
Evaluating Student Self-

Clinical setting

Assessment through Video-

Recorded Patient Simulations

Improving clinical assessment:

Clinical setting

Evaluating students' ability to

identify and apply clinical

criteria

Implementation of a Peer-

Clinical setting

Evaluation Process

\author{
Development and Clinical setting \\ implementation of an electronic \\ clinical formative assessment: \\ Dental faculty and student \\ perspectives
}
Learning experiences of oral hygiene students in the clinical environment

Clinical setting

Correlation of rater training and

Clinical setting reliability in performance assessment: Experience in a school of dentistry
Measuring the development of
Clinical setting insight by dental health professionals in training using workplace-based assessment 


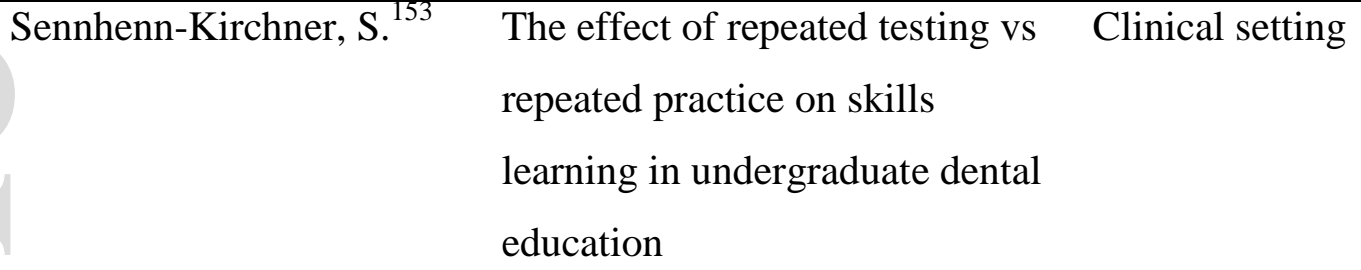

Macluskey, M. and

The retention of suturing skills Clinical setting

Hanson, C. ${ }^{154}$

in dental undergraduates

Macluskey, M. ${ }^{155}$

Development of a structured

Clinical setting

clinical operative test (SCOT) in

the assessment of practical

ability in the oral surgery

undergraduate curriculum

Moore, W. and

Bauchmoyer, S. ${ }^{156}$

Student and Faculty Perceptions Clinical setting

of Dental Hygiene Clinical

Evaluations

Walton, J.et al ${ }^{157}$

Student ePortfolios to develop

Clinical setting reflective skills and demonstrate competency development:

Evaluation of a curriculum pilot project

Weinlander, G. ${ }^{158}$

Period end clinical evaluation

Clinical setting

Abdulhadi, L. and

Mohammed, H. ${ }^{159}$

Six years' results of partially

Clinical setting

edentulous patients' treatment in

academic institute

Behere, $\mathrm{R}^{160}$

Introduction of mini-CEX in

Clinical setting

undergraduate dental education

in India

This article is protected by copyright. All rights reserved 
Bookhan, V.et al ${ }^{161}$
Criteria referenced student self- Clinical setting assessment in restorative dentistry

Bowers, $\mathrm{J}$ and Wilson, $\mathrm{J}^{162}$

Graduates' perceptions of selfassessment training in clinical dental hygiene education

Bridges, S. et $\mathrm{al}^{163}$

Bush, $\mathrm{H}$ et $\mathrm{al}^{164}$

Cairns, A et al ${ }^{165}$

Chadwick, $\mathrm{R}^{166}$

Dahllöf, G.et al ${ }^{167}$
Clinical Assessment Judgements

Clinical setting and 'Connoisseurship': Surfacing Curriculum-Wide Standards

Through Transdisciplinary

Dialogue

Failing to fail: clinicians' experience of assessing underperforming dental students

Evaluation of a pilot peer observation of teaching scheme for chair-side tutors at Glasgow University Dental School

Maximizing the clinical Clinical setting exposure of dental students to conservative dentistry: design and evaluation of a novel system A logbook for continuous selfClinical setting assessment during 1 year in paediatric dentistry
Clinical setting

Clinical setting

Clinical setting 


\begin{tabular}{|c|c|c|}
\hline Devlin, $\mathrm{H}^{168}$ & $\begin{array}{l}\text { Students' perception of the } \\
\text { prognosis for a single-surface } \\
\text { amalgam restoration }\end{array}$ & Clinical setting \\
\hline Evans, A et al ${ }^{169}$ & $\begin{array}{l}\text { The influence of self-deception } \\
\text { and impression management } \\
\text { upon self-assessment in oral } \\
\text { surgery }\end{array}$ & Clinical setting \\
\hline Evans, A et al ${ }^{170}$ & $\begin{array}{l}\text { Reliability of peer and self- } \\
\text { assessment scores compared } \\
\text { with trainers' scores following } \\
\text { third molar surgery }\end{array}$ & Clinical setting \\
\hline Evans, A et al ${ }^{171}$ & $\begin{array}{l}\text { Assessment of surgeon } \\
\text { competency to remove } \\
\text { mandibular third molar teeth }\end{array}$ & Clinical setting \\
\hline Hunter, $\mathrm{H}$ et $\mathrm{al}^{172}$ & $\begin{array}{l}\text { Deriving clinical performance } \\
\text { standards }\end{array}$ & Clinical setting \\
\hline Ihm, J and Seo, D. ${ }^{173}$ & $\begin{array}{l}\text { Does reflective learning with } \\
\text { feedback improve dental } \\
\text { students' self-perceived } \\
\text { competence in clinical } \\
\text { preparedness? }\end{array}$ & Clinical setting \\
\hline Mackenzie, $\mathrm{R}^{174}$ & $\begin{array}{l}\text { Factors essential to evaluation of } \\
\text { clinical performance }\end{array}$ & Clinical setting \\
\hline Mackenzie, R et al ${ }^{175}$ & $\begin{array}{l}\text { Analysis of disagreement in the } \\
\text { evaluation of clinical products }\end{array}$ & Clinical setting \\
\hline
\end{tabular}




\begin{tabular}{|c|c|c|}
\hline Manogue et al ${ }^{176}$ & $\begin{array}{l}\text { Clinical assessment of dental } \\
\text { students: Values and practices of } \\
\text { teachers in restorative dentistry }\end{array}$ & Clinical setting \\
\hline Metz, M et al ${ }^{177}$ & $\begin{array}{l}\text { Student Self-Assessment of } \\
\text { Operative Dentistry } \\
\text { Experiences: A Time-Dependent } \\
\text { Exercise in Self-Directed } \\
\text { Learning }\end{array}$ & Clinical setting \\
\hline Napankangas, R. et al ${ }^{178}$ & $\begin{array}{l}\text { Can the results of the OSCE } \\
\text { predict the results of clinical } \\
\text { assessment in dental education? }\end{array}$ & Clinical setting \\
\hline Navickis $\mathrm{M}$ et al ${ }^{179}$ & $\begin{array}{l}\text { Examining clinical assessment } \\
\text { practices in US dental hygiene } \\
\text { programs }\end{array}$ & Clinical setting \\
\hline Scott, B et al ${ }^{180}$ & $\begin{array}{l}\text { An investigation into the use of } \\
\text { a structured clinical operative } \\
\text { test for the assessment of a } \\
\text { clinical skill }\end{array}$ & Clinical setting \\
\hline $\begin{array}{l}\text { Van der Sanden, W et al } \\
181\end{array}$ & $\begin{array}{l}\text { Effectiveness of clinical practice } \\
\text { guideline implementation on } \\
\text { lower third molar management } \\
\text { in improving clinical decision- } \\
\text { making: a randomized controlled } \\
\text { trial. }\end{array}$ & Clinical setting \\
\hline Vernazza, $C$ et al ${ }^{182}$ & $\begin{array}{l}\text { Introduction of an e-portfolio in } \\
\text { clinical dentistry: Staff and } \\
\text { student views }\end{array}$ & Clinical setting \\
\hline
\end{tabular}

This article is protected by copyright. All rights reserved 
Wanigasooriya, $\mathrm{N}^{183}$
Student self-assessment of
Clinical setting
essential skills in dental surgery

\section{References:}

1. Roque MV, Morales I, Segarra I. Assessment of critical analysis competency in first year dentistry students using a self-evaluation tool. In: Chova LG, Martinez AL, Torres IC, eds. Inted2014: 8th International Technology, Education and Development Conference.2014:7459-7464.

2. Best HA, Messer LB. Effectiveness of interventions to promote continuing professional development for dentists. Eur J Dent Educ. 2003;7(4):147-147.

3. Mattheos N, Nattestad A, Christersson C, Jansson H, Attström R. The effects of an interactive software application on the self-assessment ability of dental students. Eur J Dent Educ. 2004;8(3):97-104.

4. Moni RW, Beswick E, Moni KB. Using student feedback to construct an assessment rubric for a concept map in physiology. Adv Physiol Educ. 2005;29(4):197-203.

5. Zijlstra-Shaw S, Kropmans TJB, Tams J. Assessment of professional behaviour - A comparison of self-assessment by first year dental students and assessment by staff. Br Dent J. 2005;198(3):165-171.

6. Broder HL, Janal M. Promoting interpersonal skills and cultural sensitivity among dental students. J Dent Educ. 2006;70(4):409-416.

7. Keselyak NT, Saylor CD, Simmer-Beck ML, Bray KK. Evaluation of Group Assessment in a Didactic Dental Hygiene Course. J Dent Hyg. 2007;81(4):87-87.

8. Cox MJ, Schleyer T, Johnson LA, Eaton KA, Reynolds PA. Making a mark--taking assessment to technology. Br Dent J. 2008;205(1):33-39.

This article is protected by copyright. All rights reserved 
9. González JA, Jover L, Cobo E, Muñoz P. A web-based learning tool improves student performance in statistics: A randomized masked trial. Computers \& Education. 2010;55(2):704-713.

10. Hay DB, Tan PL, Whaites E. Non-traditional learners in higher education: comparison of a traditional MCQ examination with concept mapping to assess learning in a dental radiological science course. Assess Eval High Edu. 2010;35(5):577-595.

11. Matthews DC. Multifaceted strategy needed to improve dentists' adherence to evidencebased guidelines. Evidence-Based Dentistry. 2010;11(2):40-40.

12. Grieveson B, Kirton JA, Palmer NOA, Balmer MC. Evaluation of workplace based assessment tools in dental foundation training. Br Dent J. 2011;211(4):E8-E8.

13. Holmes RD, Waterhouse PJ, Maguire A, et al. Developing an assessment in dental public health for clinical undergraduates attending a primary dental care outreach programme. Eur J Dent Educ. 2011;15(1):19-25.

14. Jackson SC, Murff EJT. Effectively teaching self-assessment: preparing the dental hygiene student to provide quality care. J Dent Educ. 2011;75(2):169-179.

15. Barnes E, Howells EP, Marshall K, Bullock A, Cowpe J, Thomas H. Development of the Maturity Matrix Dentistry (MMD): A primary care dental team development tool. Br Dent J. 2012;212(12):583-587.

16. Edo MM, Leache $\mathrm{EB}$, Martin $\mathrm{C}$, et al. Web-based learning in undergraduate dental education: Bite-wing X-rays in pediatric dentistry. In: Chova LG, Martinez AL, Torres IC, eds. Inted2012: International Technology, Education and Development Conference.2012:4426-4431.

17. Priya M, Muthu MS, Amarlal D, Thomas E. Continuous Assessment of Undergraduate Students at a Dental College in India. J Dent Educ. 2012;76(4):501-508.

18. Sopka S, Biermann $\mathrm{H}$, Druener S, et al. Practical skills training influences knowledge and attitude of dental students towards emergency medical care. Eur J Dent Educ. 2012;16(3):179-186.

19. Zijlstra-Shaw S, Robinson PG, Roberts T. Assessing professionalism within dental education; the need for a definition. Eur J Dent Educ. 2012;16(1):E128-E136.

20. Haj-Ali R, Al Quran F. Team-based learning in a preclinical removable denture prosthesis module in a United Arab Emirates dental school. J Dent Educ. 2013;77(3):351-357.

21. Jonas-Dwyer DRD, Abbott PV, Boyd N. First reflections: third-year dentistry students' introduction to reflective practice. Eur J Dent Educ. 2013;17(1):e64-e69.

This article is protected by copyright. All rights reserved 
22. Winning TA, Kinnell A, Wener ME, Mazurat N, J Schönwetter D. Validity of scores from communication skills instruments for patients and their dental student-clinicians. Eur J Dent Educ. 2013;17(2):93-100.

23. Gatumu MK, MacMillan FM, Langton PD, Headley PM, Harris JR. Evaluation of usage of virtual microscopy for the study of histology in the medical, dental, and veterinary undergraduate programs of a UK University. Anatomical sciences education. 2014;7(5):389398.

24. Leisnert L. Self-directed learning, teamwork, holistic view and oral health: Malmö University Electronic Publishing, Malmö University, Faculty of Odontology; 2014.

25. Prior M, Elouafkaoui P, Elders A, et al. Evaluating an audit and feedback intervention for reducing antibiotic prescribing behaviour in general dental practice (the RAPiD trial): A partial factorial cluster randomised trial protocol. Implementation Science. 2014;9(1):50-50.

26. Sampaio-Maia B, Maia JS, Leitao S, Amaral M, Vieira-Marques P. Wiki as a tool for Microbiology teaching, learning and assessment. Eur J Dent Educ. 2014;18(2):91-97.

27. Yoshida T, Ogawa T, Taguchi N, et al. Effectiveness of a simulated patient training programme based on trainee response accuracy and appropriateness of feedback. Eur J Dent Educ. 2014;18(4):241-251.

28. Al-Ansari AA, El Tautawi MMA. Comparison of Three Evidence-Based Practice Learning Assessment Methods in Dental Curricula. J Dent Educ. 2015;79(2):146-156.

29. Al-Khotani A, Björnsson O, Naimi-Akbar A, Christidis N, Alstergren P, Björnsson O. Study on self-assessment regarding knowledge of temporomandibular disorders in children/adolescents by Swedish and Saudi Arabian dentists. Acta Odontol Scand. 2015;73(7):522-529.

30. Al-Rawi W, Easterling L, Edwards PC. Development of a mobile device optimized cross platform-compatible oral pathology and radiology spaced repetition system for dental education. J Dent Educ. 2015;79(4):439-447.

31. Alfaris $\mathrm{E}, \mathrm{Naeem} \mathrm{N}$, Irfan F, et al. A one-day dental faculty workshop in writing multiplechoice questions: An impact evaluation. J Dent Educ. 2015;79(11):1305-1313.

32. El Tantawi MMA, Abdelsalam MM, Mourady AM, Elrifae IMB. E-Assessment in a limitedresources dental school using an open-source learning management system. J Dent Educ. 2015;79(5):571-583.

33. Sanchez-Sanhueza G, Cisterna Cabrera F. Praxis teaching in the ambit of learning assessment of endodontics in a Chilean university. Brazilian Oral Research. 2015;29(1).

This article is protected by copyright. All rights reserved 
34. Freda NM, Lipp MJ. Test-Enhanced Learning in Competence Based Predoctoral Orthodontics: A Four-Year Study. J Dent Educ. 2016;80(3):348-354.

35. van Schaik SM, Regehr G, Eva KW, Irby DM, O'Sullivan PS. Perceptions of Peer-to-Peer Interprofessional Feedback Among Students in the Health Professions. Acad Med. 2016;91(6):807-812.

36. Wong E, Leslie JJ, Soon JA, Norman WV. Measuring interprofessional competencies and attitudes among health professional students creating family planning virtual patient cases. BMC Med Educ. 2016;16.

37. Arias A, Peters OA, Broyles IL. New curricular design in biostatistics to prepare residents for an evidence-based practice and lifelong learning education: a pilot approach. Int Endod J. 2017;50(10):999-1010.

38. Hong B, Plugge E. Critical appraisal skills teaching in UK dental schools. Br Dent J. 2017;222(3):209-213.

39. Lipp MJ, Cho K, Kim HS. Types of Feedback in Competency-Based Predoctoral Orthodontics: Effects on Students' Attitudes and Confidence. J Dent Educ. 2017;81(5):582-589.

40. Partido BB. Dental Hygiene Students' Self-Assessment of Ergonomics Utilizing Photography. J Dent Educ. 2017;81(10):1194-1202.

41. Zijlstra-Shaw S, Roberts T, Robinson PG. Evaluation of an assessment system for professionalism amongst dental students. Eur J Dent Educ. 2017;21(4):e89-e100.

42. Morales I, Roque MV, Segarra I. Validation of self-assessment tool to measure critical analysis competency in first year dentistry students. In: Chova LG, Martinez AL, Torres IC, eds. Edulearn16: 8th International Conference on Education and New Learning Technologies. 2016:1068-1072.

43. Gonzalez-Cabezas C, Anderson OS, Wright MC, Fontana M. Association between dental student- developed exam questions and learning at higher cognitive levels. J Dent Educ. 2015;79(11):1295-1304.

44. Öcek Z, Sahin H, Baksi G, Apaydin S. Development of a rational antibiotic usage course for dentists. Eur J Dent Educ. 2008;12(1):41-47.

45. Asadoorian J, Batty HP. An evidence-based model of effective self-assessment for directing professional learning. J Dent Educ. 2005;69(12):1315-1323.

46. Clark JD, Robertson LJ, Harden RM, Laidlaw JM, McManus NK. 'On Track' - An educational resource to support dental SHO training. BDJ. 2004;197(s5):17-23.

47. Davenport ES, Fry H, Pee B, Woodman T. Learning throughout life: can a progress file help? Br Dent J. 2003;195(2):101-105.

This article is protected by copyright. All rights reserved 
48. Kournetas $\mathrm{N}$, Jaeger $\mathrm{B}, \mathrm{Axmann} \mathrm{D}$, et al. Assessing the reliability of a digital preparation assistant system used in dental education. J Dent Educ. 2004;68(12):1228-1234.

49. Haj-Ali R, Feil P. Rater reliability: short-and long-term effects of calibration training. J Dent Educ. 2006;70(4):428-433.

50. Sharaf AA, AbdelAziz AM, El Meligy OA. Intra-and inter-examiner variability in evaluating preclinical pediatric dentistry operative procedures. J Dent Educ. 2007;71(4):540-544.

51. McAndrew M, Pierre GC. Using multiple measures to evaluate a dental faculty development program. Eur J Dent Educ. 2013;17(1):1-9.

52. Renne WG, McGill ST, Mennito AS, et al. E4D compare software: an alternative to faculty grading in dental education. J Dent Educ. 2013;77(2):168-175.

53. AlHumaid J, El Tantawi M, Al-Ansari AA, Al-Harbi FA. Agreement in Scoring Preclinical Dental Procedures: Impact on Grades and Instructor-Related Determinants. J Dent Educ. 2016;80(5):553-562 .

54. Garland KV, Newell KJ. Dental hygiene faculty calibration in the evaluation of calculus detection. J Dent Educ. 2009;73(3):383-389.

55. Schmitt L, Möltner A, Rüttermann S, Gerhardt-Szép S. Study on the Interrater Reliability of an OSPE (Objective Structured Practical Examination) - Subject to the Evaluation Mode in the Phantom Course of Operative Dentistry. J Med Educ. 2016;33(4).

56. Jenkins SM, Dummer PMH, Gilmour ASM, Edmunds DH, Hicks R, Ash P. Evaluating undergraduate preclinical operative skill; use of a glance and grade marking system. J Dent. 1998;26(8):679-684.

57. Fitzgerald JT, White CB, Gruppen LD. A longitudinal study of self-assessment accuracy. Med Educ. 2003;37(7):645-649.

58. Gordon MJ. A review of the validity and accuracy of self-assessments in health professions training. Acad Med. 1991.

59. Thammasitboon K, Sukotjo C, Howell H, Karimbux N. Problem-based learning at the Harvard school of dental medicine: Self-assessment of performance in postdoctoral training. J Dent Educ. 2007;71(8):1080-1089.

60. Pileggi R, Glickman GN. A cost-effective simulation curriculum for preclinical endodontics. Eur J Dent Educ. 2004;8(1):12-17.

61. McAndrew M, Mucciolo TW, Jahangiri L. Characteristics of effective simulation (preclinical) teachers as identified by dental students: A qualitative study. J Dent Educ. 2016;80(11):12821293.

This article is protected by copyright. All rights reserved 
62. Mays KA, Branch-Mays GL. A Systematic Review of the Use of Self-Assessment in Preclinical and Clinical Dental Education. J Dent Educ. 2016;80(8):902-913.

63. de Azevedo RDA, da Rosa WLD, da Silva AF, Correa MB, Torriani MA, Lund RG. Comparative Effectiveness of Dental Anatomy Carving Pedagogy: A Systematic Review. J Dent Educ. 2015;79(8):914-921.

64. Postma TC, White JG. Developing clinical reasoning in the classroom - analysis of the 4 C/ IDmodel. Eur J Dent Educ. 2015;19(2):74-80.

65. Mattheos N, Ucer C, Van de Velde T, Nattestad A. Assessment of knowledge and competencies related to implant dentistry in undergraduate and postgraduate university education. Eur J Dent Educ. 2009;13:55-65.

66. Firmstone VR, Elley KM, Skrybant MT, Fry-Smith A, Bayliss S, Torgerson CJ. Systematic review of the effectiveness of continuing dental professional development on learning, behavior, or patient outcomes. J Dent Educ. 2013;77:300-315.

67. Hauser AM, Bowen DM. Primer on Preclinical Instruction and Evaluation. J Dent Educ. 2009;73(3):390-398.

68. Nance ET, Lanning SK, Gunsolley JC. Dental anatomy carving computer-assisted instruction program: an assessment of student performance and perceptions. J Dent Educ. 2009;73(8):972-979.

69. Dantas AK, Shinagawa A, Deboni MCZ. Assessment of Preclinical Learning on Oral Surgery Using Three Instructional Strategies. J Dent Educ. 2010;74(11):1230-1236.

70. Boushell LW, Walter R, Phillips C. Learn-A-Prep II as a predictor of psychomotor performance in a restorative dentistry course. J Dent Educ. 2011;75(10):1362-1369.

71. Polyzois I, Claffey N, McDonald A, Hussey D, Quinn F. Can evaluation of a dental procedure at the outset of learning predict later performance at the preclinical level? A pilot study. Eur J Dent Educ. 2011;15(2):104-109.

72. Gadbury-Amyot CC, Purk JH, Williams BJ, Van Ness CJ. Using tablet technology and instructional videos to enhance preclinical dental laboratory learning. J Dent Educ. 2014;78(2):250-258.

73. Bedi R, Lo E, King NM, Chan T. The effect of pictorial criteria upon the reliability of assessments of cavity preparations. J Dent. 1987;15(5):222-224.

74. Morrow JA, Pulido MT, Smith PB, McDaniel TF, Willcox AB. Effective use of e-grading in the dental simulation clinic. J Dent Educ. 2014;78(6):829-837.

This article is protected by copyright. All rights reserved 
75. Barrero C, Duqum I, Petrola F. Dental Students' Perceived Preparedness to Treat Patients in Clinic After a Fixed Prosthodontics Course: Survey Results of a Case Study. J Dent Educ. 2015;79(4):409-416.

76. Schuster GM, Hunt RJ, Haering HJ. Effect of a Pilot Preclinical Incentive Program on Dental Students' Performance on a Clinical Competency Exam. J Dent Educ. 2017;81(1):96-100.

77. Shugars DA, May KN, Vann WF. Comprehensive evaluation in a preclinical restorative dentistry technique course. J Dent Educ. 1981;45(12):801-803.

78. Wiegman J, van de Poel F, Purdell-Lewis D. The value of an assessment system in a preclinical amalgam exercise. J Dent Educ. 1985;49:312-315.

79. Abrams R, Kelley M. Student self-evaluation in a pediatric-operative technique course. J Dent Educ. 1974;28(7):385-391.

80. Edwards WS, Morse PK, Mitchell RJ. A practical evaluation system for preclinical restorative dentistry. J Dent Educ. 1982;46(12):693-696.

81. Forehand LS, Vann W, Shugars D. Student self-evaluation in preclinical restorative dentistry. J Dent Educ. 1982;46(4):221-226.

82. Cho GC, Chee WW, Tan DT. Dental students' ability to evaluate themselves in fixed prosthodontics. J Dent Educ. 2010;74(11):1237-1242.

83. San Diego J, Newton T, Quinn B, Cox M, Woolford M. Levels of agreement between student and staff assessments of clinical skills in performing cavity preparation in artificial teeth. Eur J Dent Educ. 2014;18(1):58-64.

84. Denehy G, Fuller J. Student peer evaluation: an adjunct to preclinical laboratory evaluation. J Dent Educ. 1974;38(4):200-203.

85. Brehm T. Peer evaluation of fixed partial dentures in a preclinical course. J Dent Educ. 1972;36(2):54.

86. Satterthwaite J, Grey N. Peer-group assessment of pre-clinical operative skills in restorative dentistry and comparison with experienced assessors. Eur J Dent Educ. 2008;12(2):99-102.

87. Olson $\mathrm{P}$, Comet-Epstein S. Increasing objectivity in evaluation for a preclinical technique course. J Dent Educ. 1979;43(13):688-689.

88. Karl M, Graef F, Wichmann M, Beck N. Evaluation of tooth preparations - A comparative study between faculty members and pre-clinical students. Eur J Dent Educ. 2011;15(4):250254.

89. Cohen S, AR S. Evaluation of describing and grading preclinical technical exercises. J Dent Educ. 1980;44(9):547-549.

This article is protected by copyright. All rights reserved 
90. Bazan M, Seale N. A technique for immediate evaluation of preclinical exercises. J Dent Educ. 1982;46(12):726-728.

91. Nick DR, Clark M, Miler J, Ordelheide C, Goodacre C, Kim J. The ability of dental students and faculty to estimate the total occlusal convergence of prepared teeth. J Prosthet Dent. 2009;101(1):7-12.

92. Foley JI, Richardson GL, Drummie J. Agreement Among Dental Students, Peer Assessors, and Tutor in Assessing Students' Competence in Preclinical Skills. J Dent Educ. 2015;79(11):13201324.

93. Lee C, Asher SR, Chutinan S, Gallucci GO, Ohyama H. The Relationship Between Dental Students' Assessment Ability and Preclinical and Academic Performance in Operative Dentistry. J Dent Educ. 2017;81(3):310-317.

94. Puryer J, O'Sullivan D. An introduction to standard setting methods in dentistry. Br Dent J. 2015;219(7):355-358.

95. Marshall TA, Straub-Morarend CL, Guzmán-Armstrong S, Handoo N. Evidence-based dentistry: assessment to document progression to proficiency. Eur J Dent Educ. 2017;21(4):207-213.

96. Field JC, Cowpe JG, Walmsley AD. The Graduating European Dentist: A New Undergraduate Curriculum Framework Eur J Dent Educ. 2017;21:2-10.

97. Gerhard-Szep S, Guntsch A, Pospiech P, et al. Assessment formats in dental medicine: An overview. Gms Journal for Medical Education. 2016;33(4).

98. Feil PH. Designing preclinical instruction for psychomotor skills (I)--theoretical foundations of motor skill performance and their applications to dental education. J Dent Educ. 1994;58:806-812.

99. Chambers DW, Geissberger M. Toward a competency analysis of operative dentistry technique skills. J Dent Educ. 1997;61(10):795-803.

100. Manogue M, Kelly M, Bartakova Masaryk S, et al. 2.1 Evolving methods of assessment. Eur J Dent Educ. 2002;6(s3):53-66.

101. Knight G. Toward faculty calibration. J Dent Educ. 1997;61(12):941-946.

102. Kavadella A, Kossioni AE, Tsiklakis K, et al. Recommendations for the development of emodules for the continuing professional development of European dentists Recommendations for the development of e-modules for the continuing professional development of European dentists. Eur J Dent Educ. 2013;17:45-54.

103. Kramer GA, Albino JE, Andrieu SC, et al. Dental student assessment toolbox. J Dent Educ. 2009;73(1):12-35.

This article is protected by copyright. All rights reserved 
104. Moore U, Durham J. Invited commentary: issues with assessing competence in undergraduate dental education. Eur J Dent Educ. 2011;15(1):53-57.

105. Redwood C, Winning T, Townsend G. The missing link: self-assessment and continuing professional development. Aust Dent J. 2010;55(1):15-19.

106. Council of European Dentists. Competencies required for the practice of dentistry in the European Union. file:///Users/s2125071/Downloads/CED-DOC-2009-026-E-FIN.pdf. Published 2009. Accessed 28-2-2017, 2017.

107. Licari FW, Knight GW, Guenzel PJ. Designing evaluation forms to facilitate student learning. J Dent Educ. 2008;72(1):48-58.

108. Albino JE, Young SK, Neumann LM, et al. Assessing dental students' competence: best practice recommendations in the performance assessment literature and investigation of current practices in predoctoral dental education. J Dent Educ. 2008;72(12):1405-1435.

109. Feil PH, Gatti JJ. Validation of a motor skills performance theory with applications for dental education. J Dent Educ. 1993;57(8):628-633.

110. Rhienmora P, Haddawy P, Suebnukarn S, Dailey MN. Intelligent dental training simulator with objective skill assessment and feedback. Artif Intell Med. 2011;52(2):115-121.

111. Kateeb ET, Kamal MS, Kadamani AM, Abu Hantash RO, Abu Arqoub MM. Utilising an innovative digital software to grade pre-clinical crown preparation exercise. Eur J Dent Educ. 2017;21(4):220-227.

112. Quinn F, Keogh P, McDonald A, Hussey D. Apilot study comparingthe effectiveness of conventional training and virtual reality simulation in the skills acquisition of junior dental students. Eur J Dent Educ. 2003;7(1):13-19.

113. Callan RS, Cooper JR, Young NB, Mollica AG, Furness AR, Looney SW. Effect of employing different typodonts when using E4D Compare for dental student assessment. J Dent Educ. 2015;79(6):705-710.

114. Taylor CL, Grey NJA, Satterthwaite JD. A comparison of grades awarded by peer assessment, faculty and a digital scanning device in a pre-clinical operative skills course. Eur J Dent Educ. 2013;17(1):e16-e21.

115. Callan RS, Cooper JR, Young NB, Mollica AG, Furness AR, Looney SW. Inter- and intrarater reliability using different software versions of E4D compare in dental education. J Dent Educ. 2015;79(6):711-718.

116. Callan RS, Haywood VB, Cooper JR, Furness AR, Looney SW. The validity of using E4D compare's "\% comparison" to assess crown preparations in preclinical dental education. $J$ Dent Educ. 2015;79(12):1445-1451.

This article is protected by copyright. All rights reserved 
117. Kikuchi H, Ikeda M, Araki K. Evaluation of a virtual reality simulation system for porcelain fused to metal crown preparation at Tokyo medical and dental University. J Dent Educ. 2013;77(6):782-792.

118. Kwon SR, Restrepo-Kennedy N, Dawson DV, et al. Dental Anatomy Grading: Comparison Between Conventional Visual and a Novel Digital Assessment Technique. J Dent Educ. 2014;78(12):1655-1662.

119. Mays KA, Levine E. Dental Students' Self-Assessment of Operative Preparations Using CAD/CAM: A Preliminary Analysis. J Dent Educ. 2014;78(12):1673-1680.

120. Tiu J, Cheng E, Hung T-C, et al. Effectiveness of Crown Preparation Assessment Software As an Educational Tool in Simulation Clinic: A Pilot Study. J Dent Educ. 2016;80(8):1004-1011.

121. Zou H, Jin S, Sun J, Dai Y. A cavity preparation evaluation system in the skill assessment of dental students. J Dent Educ. 2016;80(8):930-937.

122. Esser C, Kerschbaum T, Winkelmann V, Krage T, Faber FJ. A comparison of the visual and technical assessment of preparations made by dental students. Eur J Dent Educ. 2006;10(3):157-161.

123. Koo S, Kim A, Donoff RBr, Karimbux NYa. An initial assessment of haptics in preclinical operative dentistry training. J Investig Clin Dent. 2015;6(1):69-76.

124. Yekta SS, Lampert F, Kazemi S, et al. Evaluation of new injection and cavity preparation model in local anesthesia teaching. J Dent Educ. 2013;77(1):51-57.

125. Cardoso JA, Barbosa C, Fernandes S, Silva CL, Pinho A. Reducing subjectivity in the evaluation of pre-clinical dental preparations for fixed prosthodontics using the Kavo PrepAssistant ${ }^{\circledR}$. Eur J Dent Educ. 2006;10(3):149-156.

126. Mays KA, Crisp HA, Vos P. Utilizing CAD/CAM to measure total occlusal convergence of preclinical dental students' crown preparations. J Dent Educ. 2016;80(1):100-107.

127. McPherson KR, Mennito AS, Vuthiganon J, et al. Utilizing self-assessment software to evaluate student wax-ups in dental morphology. J Dent Educ. 2015;79(6):697-704.

128. Callan RS, Palladino CL, Furness AR, Bundy EL, Ange BL. Effectiveness and feasibility of utilizing E4D technology as a teaching tool in a preclinical dental education environment. J Dent Educ. 2014;78(10):1416-1423.

129. Gottlieb R, Baechle MA, Janus C, Lanning SK. Predicting performance in technical preclinical dental courses using advanced simulation. J Dent Educ. 2017;81(1):101-109.

130. Arnetzl G, Dornhofer R. PREPassistant: a system for evaluating tooth preparations. Int J Comput Dent. 2004;7(2):187-197.

This article is protected by copyright. All rights reserved 
131. Begazo C, Feilzer A. Effectiveness of preparation guidelines for an all-ceramic restorative system. Am J Dent. 2004;17(6):437-442.

132. Jasinevicius TR, Landers M, Nelson S, Urbankova A. An evaluation of two dental simulation systems: virtual reality versus contemporary non-computer-assisted. J Dent Educ. 2004;68(11):1151-1162.

133. Wierinck E, Puttemans V, Swinnen S, Steenberghe D. Effect of augmented visual feedback from a virtual reality simulation system on manual dexterity training. Eur J Dent Educ. 2005;9(1):10-16.

134. Wierinck E, Puttemans V, van Steenberghe D. Effect of reducing frequency of augmented feedback on manual dexterity training and its retention. J Dent. 2006;34(9):641-647.

135. Wierinck E, Puttemans V, Van Steenberghe D. Effect of tutorial input in addition to augmented feedback on manual dexterity training and its retention. Eur J Dent Educ. 2006;10(1):24-31.

136. Sadid-Zadeh R, D'Angelo EH, Gambacorta J. Comparing feedback from faculty interactions and virtual assessment software in the development of psychomotor skills in preclinical fixed prosthodontics. Clinical and experimental dental research. 2018;4(5):189-195.

137. Kunkel T, Engelmeier R, Shah N. A comparison of crown preparation grading via PrepCheck versus grading by dental school instructors. Int J Comput Dent. 2018;21(4):305-311.

This article is protected by copyright. All rights reserved 
Figure 1: Systematic review flow chart of the literature search and selection process
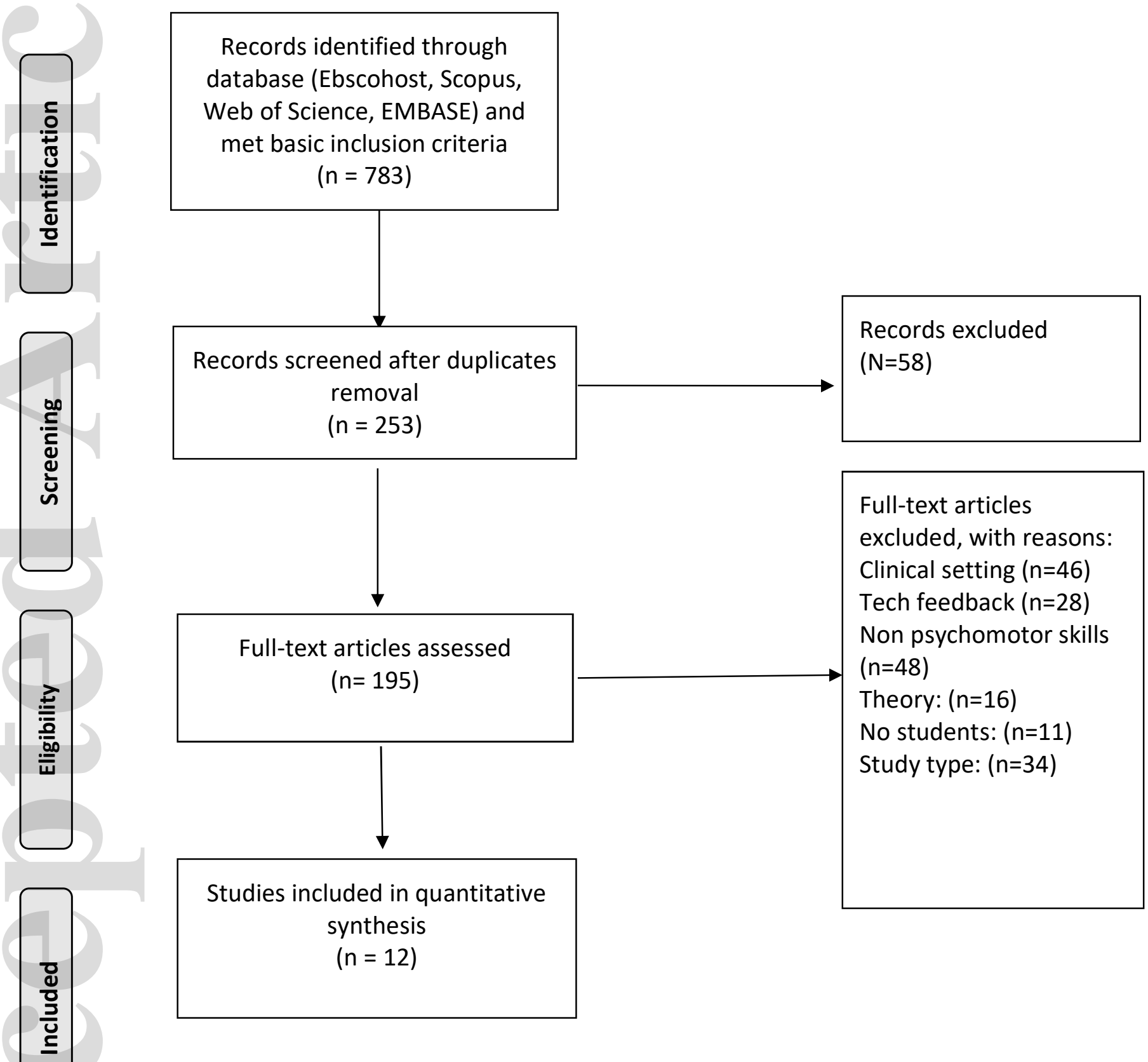

This article is protected by copyright. All rights reserved 
Figure 2: Risk of bias for Randomised Controlled Trials

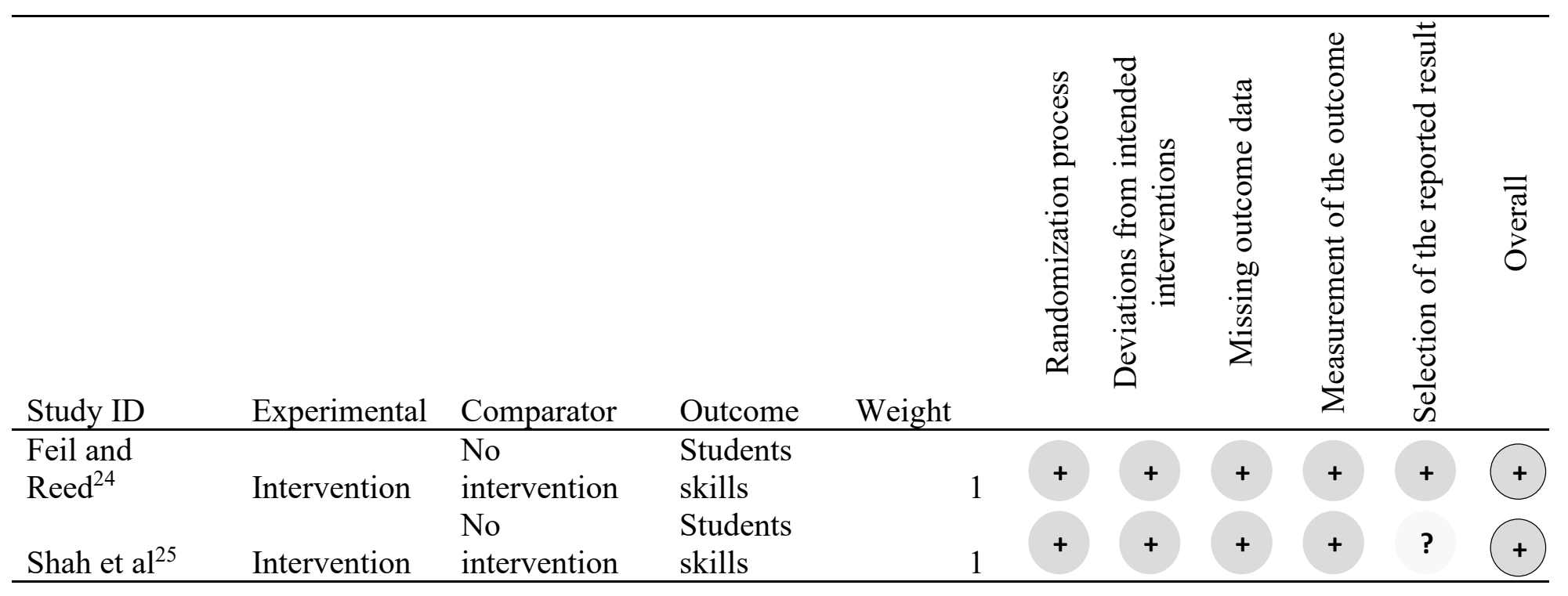

+ Low risk

? Some concerns

High risk 